$$
\begin{aligned}
& \text { की } \\
& \text { NATURE } \\
& \text { CONSERVANCY } \\
& \text { COUNCIL }
\end{aligned}
$$

\title{
World Checklist of Threatened Amphibians and Reptiles
}

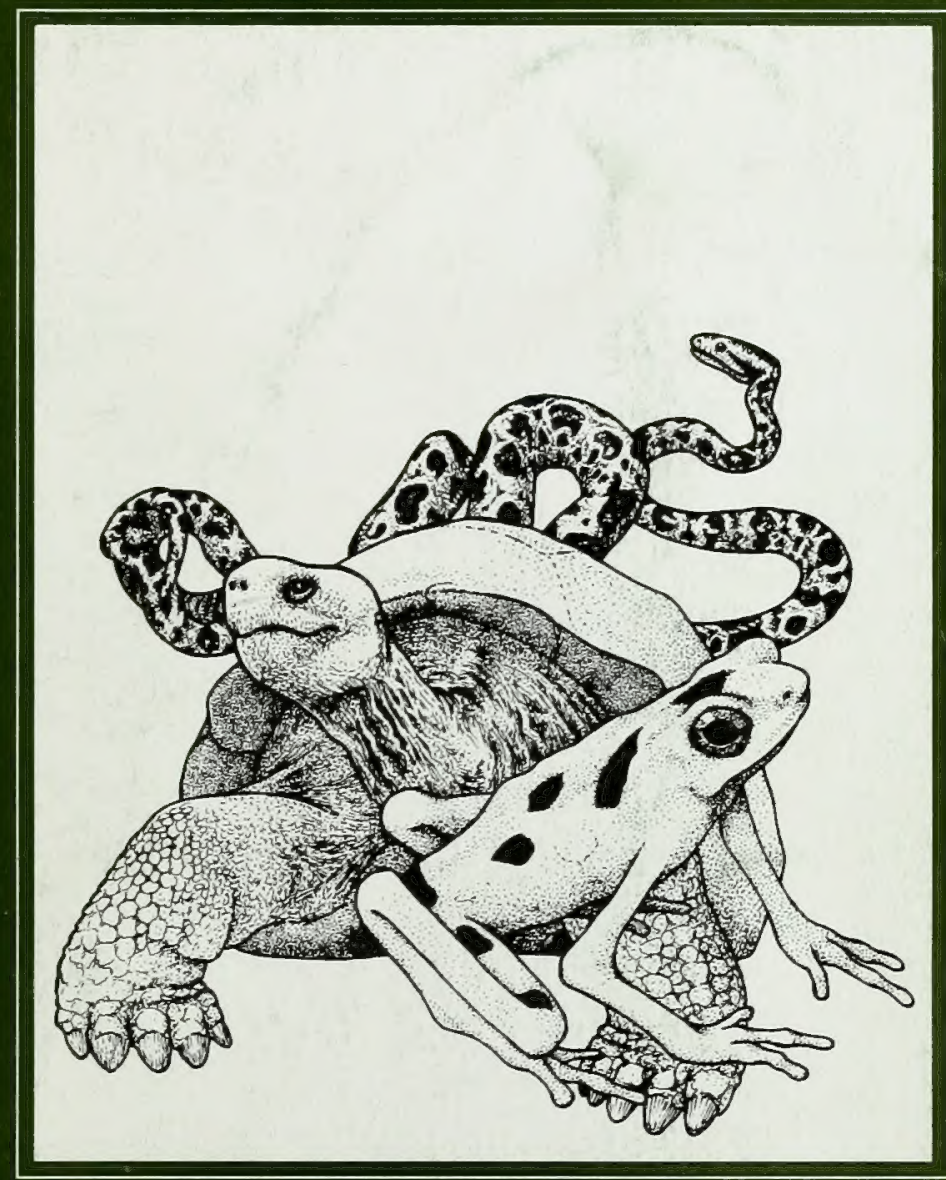

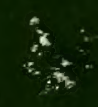


Digitized by the Internet Archive in 2010 with funding from UNEP-WCMC, Cambridge 


\section{World Checklist of Threatened Amphibiansand Reptiles}

Brian Groombridge 



\section{Contents}

Introductory Notes

Taxonomic List

AMPHIBLA

Cryptobranchidae

Ambystomatidae

Salamandridae

Plethodontidae

Proteidae

Leiopelmatidae

Pipidae

Discoglossidae

Pelobatidae

Leptodactylidae

Myobatrachidae

Bufonidae

Hylidae

Sooglossidae

Ranidae

Hyperolidae

REPTILIA

Kinosternidae

Dermatemydidae Emydidae

Testudinidae

Cheloniidae

Dermochelydidae Carettochelyidae Trionychidae
Page

Pelomedusidae

Ambystoma

Chioglossa

Batrachoseps

Eurycea

Hydromantes

Phaeognathus

Plethodon

Typhlomolge

Typhlotriton

Proteus

Leiopelma

Xenopus

Discoglossus

Pelobates

Batrachophrynus

Eleutherodactylus

Philoria

Rheobatrachus

Atelopus

Bufo

Nectophrynoides

Hyla

Pseudacris

Nesomantis

Sooglossus

Conraua

Rana

Megalixalus

Kinosternon

Sternotherus

Dermatemys

Batagur

Callagur

Clemmys

Geoclemys

Heosemys

Kachuga

Melanochelys

Morenia

Pseudemys

Rhinoclemmys

Terrapene

Chersina

Geochelone

Gopherus

Homopus

Kinixys

Malacochersus

Psammobates

Pyxis

Testudo

Caretta

Chelonia

Eretmochelys

Lepidochelys

Dermochelys

Carettochelys

Lissemys

Trionyx
Chelidae

Chelidae
Alligatoridae
Crocodylidae
Gavialidae
Sphenodontidae
Gekkonidae
Pygopodidae
Xantusidae
Agamidae
Chamaeleonidae
Iguanidae

Iguanidae

Lacertidae

Cordylidae

Teiidae

Scincidae

Anguidae

Aniellidae

Helodermatidae

Varanidae

Typhlopidae

Boidae
Page

Erymnochelys 23

Pelomedusa 23

Peltocephalus 23

Pelusios 23

Podocnemis 23

Phrynops

Platemys 24

Pseudemydura 24

Alligator 25

Caiman 25

Melanosuchus 25

Paleosuchus 25

Crocodylus 26

Osteolaemus 27

Tomistoma

Gavialis 27

Sphenodon

Cyrtodactylus 28

Oedura 28

Phelsuma 28

Paradelma 30

Xantusia 30

Hydrosaurus $\quad 30$

Uromastyx

Chamaeleo 31

Amblyrhynchus

Anolis 37

Brachylophus 37

Conolophus 37

Cyclura 38

Gambelia 38

Iguana 38

Phrynosoma 39

Sauromalus 39

Podarcis

Gallotia 39

Cordylus 39

Pseudocordylus $\quad 40$

Ameiva 40

Cnemidophorus $\quad 40$

Crocodilurus 40

Dracaena 40

Tupinambis 40

Gongylomorphus $\quad 41$

Leiolopisma 41

Macroscincus 41

Gerrhonotus 41

Aniella 41

Heloderma 41

Varanus

Typhlops 44

Acrantophis 44

Aspidites 44

Boa

Bolyeria

Calabaria

Candoia

Casarea

Charina

Chondropython

Corallus 45

Epicrates 46

Eryx 47

Eunectes 47

Exiliboa 48

Liasis 48

Lichanura 48 


\section{Acknowledgements}

$\begin{array}{clc}\text { Boidae cont. } & \text { Loxocemus } & \text { Page } \\ & \text { Python } & 49 \\ & \text { Sanzinia } & 49 \\ & \text { Trachyboa } & 50 \\ & \text { Tropidophis } & 50 \\ & \text { Ungaliophis } & 51 \\ & \text { Xenoboa } & 51 \\ \text { Colubridae } & \text { Clelia } & 52 \\ & \text { Drymarchon } & 52 \\ & \text { Elachistodon } & 52 \\ & \text { Hydrodynastes } & 52 \\ & \text { Masticophis } & 52 \\ & \text { Nerodia } & 52 \\ \text { Elapidae } & \text { Thamnophis } & 52 \\ & \text { Naja } & 53 \\ \text { Viperidae } & \text { Ogmodon } & 53 \\ & \text { Bothrops } & 53 \\ & \text { Crotalus } & 53 \\ & \text { Vipera } & 53\end{array}$

Additions and amendments 54

References 55

Index (to genera, synonyms, common, 62 and trade names)
This checklist was compiled and revised in October 1982 for the 3rd edition by Dr B

Groombridge (now at the IUCN Species

Conservation Monitoring Unit, Cambridge) at the Amphibian and Reptile Section of the British Museum (Natural History), under contract NCC/E2/36/04 from the Nature Conservancy Council of the United Kingdom.

The compiler is most grateful to the staff of the Amphibian and Reptile Section at the British Museum (Natural History), in particular to Miss A G $C$ Grandison and Dr E N Arnold, for their valuable assistance.

lst edition published 1979 as a typed document with limited circulation

ISBN $086139075 \mathrm{X}$

2nd edition published 1981

Designed by Creative Department, Manchester Ltd.

Printed for HMSO by Robendene Ltd.

ISBN 0861390954

3rd edition published 1983

Revised design by Margaret Morgan BA

ISBN 0861392248

(c) Nature Conservancy Council 1979, 1981, 1983 


\title{
Preface
}

This checklist was originally prepared to aid the United Kingdom's Scientific and Management Authorities for the Convention on International Trade in Endangered Species of Wild Fauna and Flora (CITES) which the United Kingdom has implemented since the 1 January 1976.

The aim was to provide a summary of basic data on each taxon relating to the countries where it occurs in the wild, its category in the IUCN Red Data Book, its listing in CITES and important sources of reference. Particular regard has been given to listing general names, synonyms, English vernacular names and trade names. This information will help to clarify conditions on the issue of permits to trade in amphibians and reptiles, especially in those cases where ambiguous or erroneous supporting data are supplied. It is hoped that this publication will be of value to all

Management and Scientific Authorities of the Party States to CITES and to all those concerned with the wider studies of herpetology and related matters.

This checklist covers only those amphibians and reptiles which are themselves threatened in some degree, or treated as 'look-alikes' under CITES, at the time of compilation. It is clearly desirable for the work to be extended to include the remaining taxa and therefore complete a full coverage of the group.

The present document was completed in January 1979 and since then has been subject to partial revision to take account of decisions affecting the Appendices at the 2nd meeting of the Parties to CITES at San Jose, Costa Rica in February 1979, the 3rd meeting at New Delhi in 1981 and new taxa included in Part 1 of the IUCN AmphibiaReptilia Red Data Book (1982).

The United Kingdom Scientific Authority and the Nature Conservancy Council are grateful to $\mathrm{Dr}$ Brian Groombridge for undertaking this work on their behalf.

\author{
A J B RUDGE \\ Wildlife Advisory Branch \\ Nature Conservancy Council \\ Belgrave Square \\ London SW1X 8PY \\ Winter 1983
}


The purpose of this work is to provide a combined list of the amphibian and reptile species (or subspecies) appearing on Appendices I, II, and III, of the Convention on International Trade in Endangered Species (CITES), and those included in the Red Data Book (RDB). The latter includes data sheets from the second edition issued in 1975 together with many new or revised sheets dated 1978 or 1979 (but not generally available until 1980). A useful introduction to CITES is provided by Inskipp \& Wells (1979, see Reference list C).

This revised edition incorporates changes made in the CITES Appendices at the New Delhi meeting of the Parties in 1981, and changes and additions among Testudines, Crocodylia and Rhynchocephalia made in Part 1 of the revised Amphibia-Reptilia Red Data Book (ref, 152). New and fully revised RDB accounts for remaining reptiles and for amphibians are currently in preparation, the taxa among these groups noted in this checklist are thus not fully representative.

For each taxon, the scientific name is given first, with the currently most frequently used synonyms in brackets. Common names (English language), and trade names (in brackets), appear on the following line. Very few of the listed taxa have regularly or universally used common names. In several cases, identical trade names appear to be used for different taxa

The Red Data Book taxa included are those listed in Preamble 8 (i.e., those for which full data sheets are provided) as well as those in Preamble 9 (that are suspected of being threatened, but for which full data are currently lacking).

\section{Geographical Range}

The geographical range of each taxon is given in terms of political units, listed in alphabetical order. The exceptions are some of those taxa occuring on small and/or remote islands with well-known names, that are administered by another country; for most such taxa the administering country is given in brackets, e.g. Galapagos Islands (Ecuador). In certain cases, when a taxon is found in a restricted area within a larger political unit, the name of that area is given in brackets, eg. Indonesia (West Irian)

The political names given are those in common usage, and may not in all cases be the diplomatically correct version.

When the name of a country is given as within the range of a certain species, that species may not occur throughout the country, and may even be of only peripheral occurence

Without surveying the range of each taxon in the field, it is necessary to rely on published records. Most taxonomic works give the range of a taxon in terms of broad geographical areas, rather than of precise political units. While some countries have a relatively well-known and well-reported herpetofauna, this is not true for others. These factors may occasionally have resulted in some of the geographical ranges given here being incomplete or innaccurate, although every effort has been made to counter this.

\section{Taxonomic sources.}

The most recent available checklist or revision has usually been followed for the taxonomic treatment of each species or group of species herein. Certain groups are clearly overdue for species-level revision. In the interests of stability, the present list follows the taxonomic arrangement of the RDB for the forms appearing therein, unless there is good reason to do otherwise. The genera and species are in alphabetical order within each family. The scientific and common names of North American species have been modified to accord with Standard Common and Current Scientific Names for North Ämerican Amphibians and Reptiles (Collins, Huheey, Knight, \& Smith, 1978).

The following list states the primary taxonomic source followed for most of the groups dealt with herein, and refers to an authority for each modification from that primary source. All works cited in this section appear in part (B) of the Reference list.

Caudata and Anura: follows Gorham (1974), except:

(a) Ambystoma tigninum californiense here treated as a full species, $\boldsymbol{A}$. califormiense, after Collins et al (1978).

(b) Batrachoseps aridus is added, described by Brame (1970)

(c) Family Atelopodidae no longer recognised Atelopus here assigned to family Bufonidae, after McDairmid (1969)

(d) Bufo nelsoni here reduced to a subspecies, B. boreas nelsoni, after Collins et al (1978)

(e) Five species of Nectophrynoides are added, N.cryptus and N.minutus, described by Perret (1971, 1972); also N.osgoodi (transferred from Bufo) and N.malcomi described by Grandison (1978) and N.liberiensis described by Xavier (1978)

(f) Sooglossidae here elevated to family rank (Nesomantis and Sooglossus listed), after Lynch (1973) and Duellman (1975)

(g) Rana goliath here listed as Conraua goliath, after Lamotte \& Perret (1968)

(h) Rana pipiens fisheri now in synonymy with

Rana onca, after Pace (1974).

(i) Hyperoliidae elevated to family rank

(Megalixalus listed), after Liem (1970)

(j) Myobatrachidae elevated to family rank (Philoria and Rheobatrachus listed) after Lynch (in Vial) 1973, Heyer \& Liem (1976), and others

Testudines, Crocodylia, Rhynchocephalia: follows Wermuth \& Mertens (1977), except

(a) Geoemyda tricarinata here listed as Melanochelys tricarinata, after McDowell (1964).

(b) Pseudemys terrapen malonei is treated as a full species, P.malonei, as in Schwartz \& Thomas (1975) and Pritchard (1979). The species is assigned to the genus Pseudemys rather than Chrysemys, following Wermuth \& Mertens (1977) and Pritchard (1979); there is considerable debate about the use of these names.

(c) Testudo is broken up into four additional genera (Ácinixys, Chersina, Geochelone, Psammobates) and Testudo is restricted to five Palearctic species, following Loveridge \& Williams 
(1957) and the majority of subsequent authors

(d) Podocnemis dumeriliana is listed as

Peltocephalus dumeriliana (apparently a synonym of Peltocephalus tracaxa, unpublished work noted in Pritchard (1979) and Rhodin et al) after Rhodin et al (1978)

(e) Podocnemis madagascaniensis is treated as Erymnochelys madagascariensis, after Williams (1954), Smith \& James (1958), and Rhodin et al (1978).

The above modifications within Testudines are also all incorporated in Pritchard (1979). (See Bour (1978) and Obst (1980) for different views on the nomenclature of certain of the endemic Malagasy tortoises)

(f) Crocodylus mindorensis here treated as a subspecies, C. novaeguinea mindorensis, after Wermuth \& Fuchs (1978) and several earlier authors.

Squamata; Gekkonidae and Pygopodidae: follows Wermuth (1965), except

(a) Gymnodactylus serpensinsula here treated as Cyrtodactylus serpensinsula, after Underwood (1954) and subsequent authors

(b) Phelsuma agalegae added, described by Cheke (1975)

(c) Phelsuma dubia comorensis elevated to a full species. P. comorensis, after Mertens (1966a)

(d) Phelsuma newtoni changed to $\mathbf{P}$. edwardnewtoni, following Vinson \& Vinson (1969)

(e) Phelsuma modesta added, described by Mertens (1970)

(f) Rhoptropella ocellata is treated as a species of Phelsuma, P. ocellata, after Russel (1977)

(g) Phelsuma vinsoni changed to P. ormata, see Mertens (1970)

Squamata; Agamidae: follows Wermuth (1967)

Squamata; Chamaeleontidae: follows Mertens (1966b), except

(a) Chamaeleo angeli added, described by Brygoo \& Domergue (1968)

(b) Chamaeleo antimena here treated as a full species, after Brygoo \& Domergue (1968).

(c) Chamaeleo balteatus here treated as a full species, after Brygoo \& Domerque (1968).

(d) Chamaeleo belalandaensis added described by Brygoo \& Domergue (1970)

(e) Chamaeleo capuroni added, described by Brygoo, Blanc, \& Domergue (1972).

(f) Chamaeleo eisentrauti added, described by Mertens (1968)

(g) Chamaeleo guentheri here synomised with C.pardalis, after Brygoo (1969)

(h) Chamaeleo labordi treated as a full species, after Brygoo \& Domergue (1968)

(i) Chamaeleo peyrieresi added, described by Brygoo, Blanc \& Domergue (1974).

(j) Chamaeleo tsaratananensis added,

described by Brygoo \& Domergue (1967/1968).

(k) Chamaeleo tuzetae added, described by Brygoo, Bourgat \& Domergue (1972)

(1) Also see Böhme and Klaver for further recent changes
Squamata; Iguanidae: several sources, notably

(1) Montanucci, (1970), (Crotaphytus wislizenii silus should be regarded as a full species. C. silus).

(2) Collins et al, (1978), (Crotaphytus silus assigned to Gambelia).

(3) Carey, (1975), Schwartz \& Thomas, (1975), Schwartz and Carey, (1977), (Cyclura).

(4) Peters \& Donoso-Barros, (1970), Schwartz \& Thomas, (1975), (Iguana)

(5) Gibbons, (1981), (new Brachylophus sp.).

Squamata; Lacertidae: generic assignment after Arnold, (1973)

Squamata; Cordylidae: after Wermuth, (1968)

Squamata; Teüdae: several sources, notably;

(1) Peters \& Donoso Barros, (1970)

(2) Vanzolini \& Valencia, (1965), (Dracaena)

(3) Presch, (1973), (Tupinambis, only two species recognised)

Squamata; Helodermatidae, Varanidae: follows Mertens (1963), except

(a) Varanus acanthunus primordius regarded as a full species after Cogger (1975)

(b) Varanus stomi added, described by Mertens $(1966 \mathrm{C})$

Squamata; Boidae: several sources, see notes on $\mathrm{p} 44$ and $\mathrm{p} 46$

Squamata; Colubridae: several sources, notably lelia).

1) Peters \& Orejas-Miranda, (1970), (Clelia

(2) Gans \& Williams, (1954), Fleming and Fleming, (1973), 433 (Elachistodon).

(3) Dowling \& Gibson, (1970); (Cyclagras gigas assigned to Hydrodymastes).

(4) Rossman \& Eberle, (1977); (Natrix sipedon and $\mathbf{N}$. fasciata assigned to Nerodia).

Squamata; Elapidae, Viperidae: chiefly after Klemmer, (1963) 


\section{ExplanatoryKey}

For each taxon, there are at least two entries in the four columns down the right-hand side of the page.

CITES: I, II, or III, referring to the CITES Appendix on which the taxon is listed as at June 1981

RDB: Red Data Book status categories,

$$
\begin{aligned}
& E=\text { Endangered } \\
& V=\text { Vulnerable } \\
& R=\text { Rare }
\end{aligned}
$$

$\mathrm{O}=$ Out of danger

Id = Indeterminate (given as "I" in RDB, but

modified here to avoid confusion with the

CITES "I" entry)

$\mathrm{K}$ = Insufficiently Known; suspected of being

threatened, but without full data.

Exp: Major exploitation of taxon, where known.

$\mathrm{F}=\mathrm{Food}$ (may include local and commercial use)

LA $=$ Live animal trade (primarily for the pet

trade)

Pr $=$ Preserved specimens

SK $=$ Skins, for the leather trade

TS $=$ Tortoise-shell

When known, a category of secondary importance is given in brackets.

Ref. Entry here is a number referring toanitem in part (A) of the list of References providing incidental or exhaustive information on the survival status of the taxon 


\section{Class:AMPHIBIA}

Order: CAUDATA (Urodela), Newts and Salamanders

Family: CRYPTOBRANCHIDAE

Andrias davidianus (Megalobatrachus davidianus)

Chinese Giant Salamander

China

Andrias japonicus (Megalobatrachus japonicus)

Japanese Giant Salamander

Japan

Family: AMBYSTOMATIDAE

Ambystoma californiense (A. tigrinum califormiense)

California Tiger Salamander

USA (California)

Ambystoma dumerilii

Mexico

(RDB entry is for Ad. dumerilii, the Lake Patzcuaro Salamander or Achoque. from Lake Patzcuaro, Michoacan; the remaining subspecies A.d. queretarensis, Michoacan, is not included)

Ambystoma lermaensis

Lake Lerma Salamander

Mexico

Ambystoma macrodactylum croceum

Santa Cruz Long-toed Salamander

USA (Califomia)

Ambystoma mexicanum

Axolotl

Mexico (Lake Xochimilco region)

Family: SALAMANDRIDAE

Chioglossa lusitanica

Gold-striped Salamander

Portugal, Spain

Family: PLETHODONTIDAE

\section{Batrachoseps aridus}

Desert Slender Salamander

USA (Califomia)

Batrachoseps simatus 


\section{Batrachoseps stebbinsi}

Tehachapi Slender Salamander

USA (Califomia)

\section{Eurycea nana}

San Marcos Salamander

USA (Texas)

\section{Hydromantes brunus}

Limestone Salamander

USA (Califomia)

\section{Hydromantes shastae}

Shasta Salamander

USA (California)

\section{Phaeognathus hubrichti}

Red Hills Salamander

USA (Alabama)

\section{Plethodon larselli}

Larch Mountain Salamander

USA (Oregon, Washington)

\section{Plethodon neomexicanus}

Jemez Mountains Salamander

USA (New Mexico)

\section{Plethodon nettingi (P. richmondi nettingi)}

Cheat Mountain Salamander

USA (West Virginia)

Typhlomolge rathbuni (Eurycea rathbuni)

Texas Blind Salamander

USA (Texas)

\section{Typhlotriton spelaeus}

Grotto Salamander

USA

\section{Family: PROTEIDAE}

\section{Proteus anguinus}

Olm

Yugoslavia (and introduced at Vicenza, Italy)
R $\quad 4,43,75$

- R $\quad-443$

- $R-4,43,75$

- $\mathrm{R} \quad 4,43,75$

- R - 43

- Id $\quad-4,43$

- V - 4,43,73

- $E-4,43,51$

- V LA 38,43 
Order: ANURA (Salientia)

Frogs and Toads

Family: LEIOPELMATIDAE

(LEIOPELMIDAE; Leiopelma is sometimes included in the ASCAPHIDAE)

\section{Leiopelma archeyi}

Archey's Frog, Coromandel Leiopelma

New Zealand

\section{Leiopelma hamiltoni}

Hamilton's Frog, Stephen Island Leiopelma

New Zealand

\section{Leiopelma hochstetteri}

Hochstetter's Frog, North Island Leiopelma

New Zealand

Family: PIPIDAE

\section{Xenopus gilli}

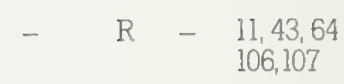

Cape Platanna, Cape Clawed Toad

South Africa (Cape Town region)

Family: DISCOGLOSSIDAE

\section{Discoglossus nigriventer}

Israel Painted Frog

Israel (Lake Huleh)

Family: PELOBATIDAE

\section{Pelobates fuscus insubricus}

Italian Spadefoot Toad

Italy (plains of River Po). Switzerland (Ticino, probably extinct)

Family: LEPTODACTYLIDAE

\section{Batrachophrynus macrostomus}

Lake Junin (Giant) Frog

Peru (Lake Junin)

\section{Eleutherodactylus jasperi}

Golden Coqui Frog

Puerto Rico

Family: MYOBATRACHIDAE

(altematively regarded as a subfamily of LEPTODACTYLIDAE)

\section{Philoria frosti}

Baw-baw Frog

Australia 


\section{Rheobatrachus silus}

Platypus Frog

Australia (Queensland)

Family: BUFONIDAE

\section{Atelopus varius zeteli}

Golden Frog, Zetek's Golden Frog

I $-\underset{(\mathrm{PY})}{\operatorname{LA} 50}$

Panama (Valle de Anton)

(Atelopus is sometimes assigned to a separate family, ATELOPODIDAE)

\section{Bufo boreas nelsoni}

Amargosa Toad

USA (Califomia, Nevada)

\section{Bufo exsul}

Black Toad, Deep Springs Toad

USA (California)

(sometimes treated as a subspecies of B. boreas, B.boreas exsul)

\section{Bufo houstonensis}

Houston Toad

USA (Texas)

\section{Bufo periglenes}

Golden Toad, Orange Toad

Costa Rica

\section{Bufo retiformis}

Sonoran Green Toad

Mexico (Sonora), USA (Arizona)

\section{Bufo superciliaris}

Cameroon Toad

Cameroon, Equatorial Guinea, Gabon, Ghana, Guinea, Nigeria

Zaire, probably also in other countries of west and west-central Africa

\section{Nectophrynoides cryptus}

Viviparous African Toad

Tanzania (Uluguru Mts.)

\section{Nectophrynoides liberiensis}

Liberia (Mt. Nimba)

\section{Nectophrynoides malcomi}

\section{Ethiopia}

\section{Nectophrynoides minutus}

Tanzania (Uluguru Mts.)

\section{Nectophrynoides occidentalis}

Mt. Nimba Viviparous Toad

Guinea (Mt. Nimba), and adjacent parts of Ivory Coast 
Nectophrynoides osgoodi

Ethiopia

Nectophrynoides tornieri

Tanzania (Magrotto and Usambara Mts.)

Nectophrynoides viviparus

Tanzania (Kinga, Rungwe, and Uluguru Mts.)

Family: HYLIDAE

Hyla andersoni

Pine Barrens Tree Frog

USA

(Florida populations noted as Endangered in RDB)

Pseudacris streckeri illinoensis

Ilinois Chorus Frog

USA

Family: SOOGLOSSIDAE

(sometimes treated as a subfamily of RANIDAE)

Nesomantis thomasseti

Thomasset's Seychelles Frog

Seychelle Is. (Mahe, Silhouette)

Sooglossus sechellensis

Seychelles Frog

Seychelle Is. (Mahe, Silhouette)

Sooglossus gardineri

Gardiner's Seychelles Frog

Seychelle Is. (Mahe, Silhouette)

Family: RANIDAE

Conraua goliath (Rana goliath, Gigantorana goliath)

Goliath Frog

Cameroon, Equatonial Guinea

\section{Rana onca (inc. Rana pipiens fisheri)}

Relict Leopard Frog (inc. Vegas Valley Leopard Frog)

USA (Arizona, Utah, Vegas Valley Leopard Frog restricted to Nevada)

Family: HYPEROLIIDAE

(sometimes treated as a subfamily of

RHACOPHORIDAE)

Megalixalus seychellensis (M. infrarufus)

Id $\quad-43$

Seychelle Islands Tree Frog

Seychelle Islands (Mahe, Praslin, La Digue, Silhouette) 
Order: TESTUDINES,

Tortoises, Turtles

Suborder: CRYPTODIRA

Family: KINOSTERNIDAE

\section{Kinosternon augustipons}

Narrow-bridged Mud Turtle

Costa Rica, Nicaragua, Panama

\section{Kinosternon creaseri}

Creaser's Mud Turtle

Mexico

\section{Kinosternon dunni}

Dunn's Mud Turtle

Columbia

Sternotherus minor depressus

Flattened Musk Turtle

USA

\section{Family: DERMATEMYDIDAE}

\section{Dermatemys mawii}

Central American River Turtle

Belize, Guatemala, Mexico

\section{Family: EMYDIDAE}

\section{Batagur baska}

Common Batagur, River Terrapin, Tuntong

Bangladesh, Burma, India, Indonesia, Kampuchea

Malaysia, Thailand, Vietnam?

\section{Callagur borneoensis}

Painted Terrapin

Indonesia (Kalimantan, Sumatra), Malaysia, Thailand

\section{Clemmys muhlenbergii}

Bog Turtle, Muhlenberg's Turtle

USA

\section{Geoclemys hamiltonii (Damonia hamiltonii)}

Black Pond Turtle, Spotted Pond Turtle

Bangladesh, India, Pakistan

\section{Heosemys silvatica}

Kavalai Forest Turtle

India (Kerala)

\section{Kachuga tecta tecta}

Indian Sawback or Tent Turtle, Roofed or Dura Turtle

Bangladesh, India, Pakistan

Melanochelys tricarinata (Geoemyda tricarinata, Nicoria tricarinata)

Three-keeled Land Tortoise 
Morenia ocellata

Burmese Swamp Turtle

Burma

Pseudemys alabamensis

Alabama Red-bellied Turtle

USA

Pseudemys felis

Cat Island Turtle

Bahamas (Cat Island)

Pseudemys malonei (Pseudemys terrapen malonei, Chrysemys malonei)

Inagua Island Turtle

Bahamas (Great Inagua Island)

Pseudemys rubriventris bangsi

Plymouth Red-bellied Turtle

USA

Pseudemys scripta callirostris (Pseudemys omata callirostris, Chrysemys ormata callirostris)

South American Red-lined Turtle

Colombia, Venezuela

\section{Rhinoclemmys areolata}

Furrowed Wood Turtle

Belize, Guatemala, Honduras, Mexico

\section{Rhinoclemmys rubida}

Mexican Spotted Wood Turtle

Mexico

\section{Terrapene coahuila}

Aquatic or Water Box Turtle

Mexico (Coahuila)

\section{Family: TESTUDINIDAE}

\section{Chersina angulata (Testudo angulata)}

Angulated or Bowsprit Tortoise

Namibia, South Africa

\section{Geochelone carbonaria (Testudo carbonaria)}

Red-foot Tortoise

Brazil, Colombia, French Guiana, Guyana,

Lesser Antilles, (several islands, probably introduced),

Panama, Paraguay, Suninam, Trinidad, Venezuela

Geochelone chilensis (inc. G.donosobarrosi and G.petersi; Testudo chilensis)

Argentine Tortoise, Chaco Tortoise

Argentina, Paraguay, probably Bolivia

Geochelone denticulata (Testudo denticulata)

Yellow-foot Tortoise, Jaboti, Forest Tortoise,

Brazilian Giant Tortoise, Hercules Tortoise 
Geochelone elegans (Testudo elegans)

Indian Starred or Star Tortoise

India, Pakistan, Sri Lanka

Geochelone elephantopus (Testudo elephantopus)

Galapagos Giant Tortoise

Galapagos Islands (Ecuador)

Geochelone elongata (Testudo elongata)

Yellow Tortoise

Bangladesh, Burma, China, India, Kampuchea, Laos, Malaysia, Nepal, Thailand, Vietnam

Geochelone emys (Testudo emys)

Burmese Brown Tortoise

Burma, India, (Assam), Indonesia, Kampuchea, Laos?,

Malaysia, Thailand, Vietnam?

Geochelone forstenii (Testudo forstenii)

Sulawesi (Celebes) Tortoise

Indonesia (Halmahera, Sulawesi)

Geochelone gigantea (Testudo gigantea)

Aldabra Giant Tortoise

Aldabra, Seychelle Islands (introduced)

Geochelone impressa (Testudo impressa)

Impressed Tortoise

Burma, China, Malaysia (peninsula), Thailand, Vietnam,

probably also Kampuchea and Laos

Geochelone pardalis (Testudo pardalis)

II

(F)

Leopard Tortoise, Mountain Tortoise

Angola, Botswana, Ethiopia, Kenya, Lesotho, Malawi

Mozambique, Namibia, Somalia, South Africa, Sudan, Swaziland,

Tanzania, Uganda, Zambia, Zimbabwe

Geochelone platynota (Testudo platynota)

Burmese Starred Tortoise

Burma

Geochelone radiata (Testudo radiata, Asterochelys radiata)

Radiated Tortoise

Madagascar

Geochelone sulcata (Testudo sulcata)

Spurred or Grooved Tortoise

Chad, Ethiopia, Mali, Mauntania, Niger, Nigeria, Senegal,

Sudan, probably also Cameroon, Central African Republic,

Djibouti, Upper Volta

Geochelone travancorica (Testudo travancorica)

Travancore Tortoise

India

Geochelone yniphora (Testudo yniphora, Asterochelys yniphora)

Madagascar Tontoise, Angonoka

Madagascar

18
II

II

II

II

LA -

E $\quad-\quad 43,114$

152

II

LA

$\begin{array}{lll}\text { K } & \text { F } & 54,152\end{array}$

II

152

141,152

48.152 
Gophenus agassizii (G. polyphemus agassizii)

Desert Tortoise, Westem Gopher Tortoise

Mexico, USA

Gopherus berlandieri (G.polyphemus berlandieri)

Texas Gopher Tortoise, Berlandier's Tortoise

Mexico, USA (Texas)

Gopherus flavomarginatus (G.polyphemus flavomarginatus)

Bolson Tortoise, Mexican Giant Gopher Tortoise

Mexico

Gophenus polyphemus

Florida Gopher Tortoise

USA

\section{Homopus areolatus}

II $\quad \mathrm{V} \quad$ LA $\quad 4.15,29$

75,144

152

II Id $F$

$L A$

152

LA 101,144

F 152

Parrot-beaked or Areolated Tortoise

South Africa

Homopus boulengeri

II

Boulengers Tortoise

Namibia, South Africa

Homopus femoralis

II

Karroo Tortoise

South Africa

Homopus signatus

II

Speckled Tortoise

Namibia, South Africa

\section{Kinixys belliana}

Bell's Hinged Tortoise

II

Angola, Benin, Botswana, Burundi?, Cameroon, Central African Republic, Congo, Djibouti, Ethiopia, Gabon, Gambia, Ghana, Guinea, Guinea Bissau, Ivory Coast?, Kenya, Lesotho?, Liberia, Malawi, Mali, Mozambicue, Namibia, Nigeria, Rwanda, Senegal, Sierra Leone, Somalia, South Africa, Sudan, Swaziland, Tanzania, Togo, Uganda, Zaire, Zambia, Zimbabwe, (introduced on Madagascar)

\section{Kinixys erosa}

Schweigger's, Common, or Forest Hinged Tortoise

Angola, Benin, Cameroon, Central African Republic, Congo Equatorial Guinea, Gabon, Gambia, Ghana, Guinea, Ivory Coast, Liberia, Nigena, Senegal, Sierra Leone, Togo, Uganda, Upper Volta, Zaire, (introduced on Madagascar)

\section{Kinixys homeana}

Home's Hinged Tortoise

Benin, Cameroon, Congo, Equatorial Guinea, Gabon, Ghana, Guinea, Ivory Coast, Liberia, Nigeria, Togo, Zaire

\section{Malacochersus tornieri}

Pancake Tortoise

Kenya, Tanzania 
Psammobates geometricus (Testudo geometrica)

Geometric Tortoise

South Africa

\section{Psammobates oculifer (Testudo oculifera)}

Serrated Tortoise, Toothed Cape Tortoise, Kuhl's Tortoise Botswana, Namibia, South Africa

Psammobates tentorius (Testudo tentoria)

Tent Tortoise

Namibia, South Africa

Pyxis arachnoides

Spider Tortoise

Madagascar

Pyxis planicauda (Testudo planicauda, Acinixys planicauda)

Madagascar Flat-shelled Tortoise

Madagascar

\section{Testudo graeca}

Spur-thighed, Common, or Greek Tortoise

Albania, Algeria, Bulgania, Egypt, Greece, Iran, Iraq, Israel, Italy (including Sardinia and Sicily, probably introduced), Jordan, Lebanon, Libya, Malta, Morocco, Romania, Spain (inc Balearic Islands), Syria, Tunisia, Turkey, USSR, Yugoslavia, (introduced in southem France)

(1. RDB entry is for T.graeca graeca) from Algeria, Libya, Morocco, Spain, Tunisia, and introduced in France

2. Populations in eastern Iran are occasionally treated as a full species, T.zarudnyi)

\section{Testudo hermanni}

Hermann's Tortoise, Greek Tortoise

Albania, Bulgania, France (inc. Corsica), Greece, Italy (inc. Sardinia, Sicily), Romania, Spain (Balearic Islands, also reported from mainland), Turkey, Yugoslavia

\section{Testudo horsfieldii (Agrionemys horsfieldii)}

Horsfield's Tortoise, Afghan Tortoise

Afghanistan, China, Iran, Pakistan, USSR

\section{Testudo kleinmanni}

Egyptian Tortoise

Egypt, Israel, Libya

\section{Testudo marginata}

Margined Tortoise

Greece, Italy (Sardinia, introduced, possibly mainland also)

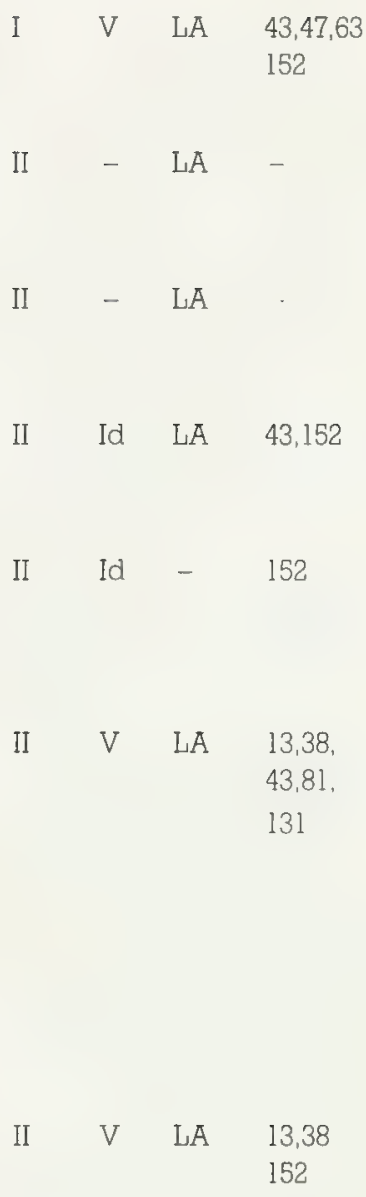




\section{Family: CHELONIIDAE}

(Because of the wide and somewhat unpredictable distribution of sea turties away from the nesting beaches, it is impractical to list those countries in whose territorial waters individual sea turtles may be expected to occur. This applies to members of the CHELONIIDAE and DERMOCHELYDIDAE.)

\section{Caretta caretta}

Loggerhead Turtle

Nesting recorded on beaches of tropical, subtropical, and temperate seas (Atlantic, Indian, Mediterranean, Pacific), wandering individuals recorded in temperate and arctic waters (to $70^{\circ} \mathrm{N}$ at Murmansk, USSR, and to $35^{\circ} \mathrm{S}$ at Rio de la Plata, Argentina)

\section{Chelonia depressa}

Flatback Turtle

Nests in north and north east Australia, not recorded outside adjacent waters

\section{Chelonia mydas}

Green Turtle

Nesting recorded on beaches of tropical and subtropical seas (Atlantic, Indian, Mediterranean, Pacific), wandering individuals recorded in temperate waters (to ca. $52^{\circ} \mathrm{N}$ in English Channel, and to $43^{\circ} \mathrm{S}$ in Chile). Major breeding and feeding areas within $20^{\circ} \mathrm{N}$ to $20^{\circ} \mathrm{S}$.

Breeding populations in East Pacific sometimes regarded as a full species Chelonia agassizii.

\section{Eretmochelys imbricata}

Hawksbill Turtle

Nesting recorded at scattered localities on beaches of tropical and subtropical seas, usually between $25^{\circ} \mathrm{N}$ and $25^{\circ} \mathrm{S}$ (Atlantic Indian, Pacific, rarely or formerly in Mediterranean), wandering individuals occasionally recorded in temperate regions.

\section{Lepidochelys kempii}

Kemp's Ridley, Atlantic Ridley

Single major nesting site is in Mexico (Rancho Nuevo, on Gulf coast of State of Tamaulipas), occurs most frequently in Gulf of Mexico, wandering individuals recorded in Atlantic waters (to $58^{\circ} \mathrm{N}$ in Scotiand), and very occasionally in Mediterranean

\section{Lepidochelys olivacea}

Olive Ridley, Pacific Ridley

Nesting recorded on beaches of tropical and subtropical seas (Atlantic, Indian, Pacific), most sites between $30^{\circ} \mathrm{N}$ and $20^{\circ} \mathrm{S}$ occasionally recorded in the Caribbean region although does not appear to breed there

$\begin{array}{cl}\mathrm{I} \quad \mathrm{F} \quad & 4,6,25,38 \\ & 40,41,43, \\ & 54,68,69 \\ & 103,123 \\ & 126,133 \\ & 139,152\end{array}$

$41,43,130$ 152

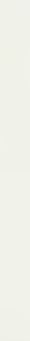
E F $\quad 4,6,18,25$
(SK) $33,37,38$,
$40,41,43$
$54,62,65$
$68,69,88$,
99,114
117.120
124,130
133,139
146,152
E $F \quad 4,6,22,25$
TS 30,33,38,
(SK) $39,40,41$,
$43,54,62$,
$68,69,120$
128,130
133,139
146.152

E

F $\quad 120,128$,

(SK) 130,133 ,

139,146

$4,6,23,25$,

$38,41,43$

$69,91,133$ 152 $65,68,90$ 91,121 130,139 146,152 
Family: DERMOCHELYDIDAE

(DERMOCHELYIDAE)

(see note under Family: CHELONIIDAE, p21)

\section{Dermochelys coriacea}

Leatherback, Leathery Turtle, Luth

Nesting recorded on beaches of tropical and subtropical seas

(Atlantic, Indian, Mediterranean, Pacific), most sites between $30^{\circ} \mathrm{N}$ and $20^{\circ} \mathrm{S}$, wandering individuals recorded in temperate waters

(north to Iceland and Norway)

$\begin{array}{cc}\mathrm{E} \quad \mathrm{F} \quad & 4,6,25,38, \\ & 40,41,43, \\ & 54,57,62 \\ & 68,69,70 \\ & 87,116 \\ & 121,122 \\ & 130,132, \\ & 133,146 \\ & 152\end{array}$

\section{Family: CARETTOCHELYIDAE}

\section{Carettochelys insculpta}

New Guinea Plateless Turtle

Australia (Northem Territory), Indonesia (Irian Jaya),

Papua New Guinea

\section{Family: TRIONYCHIDAE}

\section{Lissemys punctata andersoni (L.p.punctata)}

Indian Flap-shell Turtle

Bangladesh, Burma (Akyab), India, Nepal, Pakistan

\section{Trionyx ater}

Cuatro Cienegas or Black Soft-shell Turtle

Mexico

\section{Trionyx gangeticus}

Indian or Ganges Soft-shell Turtle

Afghanistan, Bangladesh, India, Nepal, Pakistan

\section{Trionyx hurum}

Peacock-marked Soft-shell Turtle

Bangladesh, India

\section{Trionyx nigricans}

Dark Soft-shell Turtle

Bangladesh

\section{Trionyx triunguis}

Nile Soft-shell Turtle

Angola (inc. Cabinda, and south to Cunene River on AngolaNamibia border), Cameroon, Chad, Egypt, Ethiopia, Gabon, Gambia, Ghana, Guinea Bissau, Israel, Kenya, Liberia, Niger, Nigeria, Senegal, Sierra Leone, Somalia, Sudan, Togo, Turkey (south), Uganda, Zaire, probably also Benin, Central African Republic, Congo, Djibouti, Equatorial Guinea, Guinea,

Ivory Coast, Mali, and Upper Volta 


\section{Suborder: PLEURODIRA, Side-necked Turtles}

\section{Family: PELOMEDUSIDAE}

\section{Erymnochelys madagascariensis (Podocnemis madagascariensis)}

\section{Madagascar}

(Untll recently (see note on p8), a majority of taxonomists have assigned this species, and Peltocephalus dumeriliana, to the genus Podocnemis. All species of Podocnemis are listed on CITES Appendux II. It is here assumed, pending discussion of the matter that the intention was to include these two species also regardless of a change in genenc assignment)

\section{Pelomedusa subrufa}

III

Helmeted Turtle

Angola, Benin, Botswana, Cameroon, Ethiopia, Ghana, Kenya, Madagascar, Malawi, Mali, Mauritania, Mozambique, Namibia, Nigeria, Saudi Arabia (southwest), Senegal, Somalia, South Africa, Sudan, Tanzania, Togo, Uganda, North Yemen, Zaire, Zambia, Zimbabwe, probably also Central African Republic, Chad, Congo Djibouti, Equatorial Guinea, Gambia, Guinea, Guinea Bassau, Ivory Coast, Liberia, Niger, Upper Volta

\section{Peltocephalus dumeriliana (Peltocephalus tracaxa,} new name; Podocnemis dumeriliana)

Brazil, Colombia, Ecuador? French Guiana? Peru, Venezuela (see note under Erymnochelys madagascariensis, above)

\section{Pelusios spp.}

Ghana has proposed that all species of Pelusios found in Ghana be placed on Appendix III, the following four species, of the eight currently recognised by Wermuth \& Mertens (1977), may be expected to occur in Ghana. The systematics of this genus is subject to some dispute

\section{Pelusios adansonii}

White-breasted Side-necked Turtle, Adanson's Turtle

III

(Ghana)

III

(Ghana)

Angola, Burundi, Congo, Gambia, Guinea, Liberia, Madagascar, Malawi, Rhodesia, Rwanda, Senegal, Seychelle Islands, Sierra Leone, Tanzania, Zaire, Zambia, possibly Ghana

\section{Pelusios gabonensis}

Stripe-backed Side-necked Turtle, Gaboon Turtle West Africa (from Liberia east, inc. Ghana, to Gabon)

\section{Pelusios niger}

Black Side-necked Turtle

West Africa (from Gambia east, possibly inc. Ghana, to Āngola)

Podocnemis erythrocephala (Podocnemis cayennensis)

Irapuca, Red-headed Amazon Turtle

Brazil, Venezuela, perhaps Colombia 


\section{Podocnemis expansa}

Arrau, South American River Turtle

Bolivia, Brazil, Colombia, Ecuador?, Guyana, Peru, Venezuela, perhaps French Guiana and Surinam

\section{Podocnemis lewyana}

Magdalena River Turtle

Colombia

\section{Podocnemis sextuberculata}

Brazil, Colombia, Peru

\section{Podocnemis unifilis}

Terecay, Tracajá, Yellow-spotted Amazon Turtle

Bolivia, Brazil, Colombia, Ecuador, French Guiana, Guyana, Peru, Surinam, Venezuela

\section{Podocnemis vogli}

Colombia, Venezuela

\section{Family: CHELIDAE}

\section{Phrynops dahli}

Dahl's Toad-headed Turtle

Colombia (Bolivar)

\section{Phrynops hogei}

Hoge's Sideneck Turtle

Brazil

\section{Phrynops rufipes}

Red-headed Sideneck Turtle

Brazil, Colombia

\section{Platemys pallidipectoris}

Chaco Sideneck Turtle

Argentina, Bolivia?, Paraguay

\section{Platemys spixii}

Spix's Sideneck Turtle

Argentina, Brazil, Uruguay

\section{Pseudemydura umbrina}

Short-necked or Western Swamp Turtle

Australia (Bullsbrook)

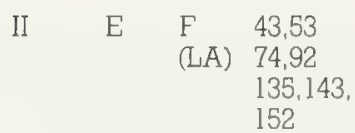

II $\quad$ E $\quad F \quad 43,53$

(LA) 74,92

135,143,

152

II Id $F \quad 53,135$

(LA) 152

II $\quad \mathrm{K} \quad F \quad 53,135$

143,152
$\begin{array}{llll}\text { II } & \text { V } & \text { F } & 92.119\end{array}$
(LA) 135,143
152

II $\quad-\quad F \quad 53,135$ 


\section{Order: CROCODYLIA}

For the many and vanable trade names of Crocodylia, see King \& Brazaits 1971, and Wermuth \& Fuchs, 1978, (See Reference lıst C.p61)

\section{Family: ALLIGATORIDAE}

\section{Alligator mississippiensis}

American Alligator

USA

\section{Alligator sinensis}

Chinese Alligator

China

\section{Caiman crocodilus sub-spp.}

Caiman crocodilus apaporiensis

(C.sclerops apaporiensis)

Rio Apaporis (Spectacled) Caiman

Colombia

\section{Caiman crocodilus (C.sclerops)}

3 to 5 remaining subspecies

Spectacled Caiman

Argentina, Bolivia, Brazil, Colombia, Costa Rica, Ecuador, French Guiana, Guatemala, Guyana, Honduras, Mexico, Nicaragua, Panama, Paraguay, Peru, El Salvador, Surinam, Trinidad \& Tobago, Uruguay, Venezuela (C.c.yacare, the Jacaré, from Argentina, Bolivia, Brazil, Paraguay, Unuguay, is sometimes treated as a full species)

(In Part 1 of the new Amphibia-Reptila RDB (ref. 152) the three widely recognised subspecies are categorised as follows. C.C. crocodilus ' $V$ '; C.c. fuscus ' $V$ ' and C.c. yacare 'Id')

\section{Caiman latirostris}

Broad-nosed Caiman

Argentina, Brazil, Paraguay, Unuguay

\section{Melanosuchus niger}

\section{Black Caiman}

Bolivia, Brazil, Colombia, Ecuador, French Guiana, Guyana,

\section{Paraguay, Peru}

\section{Paleosuchus palpebrosus}

Dwarf Caiman, Cuvier's Smooth-fronted Caiman

Brazil, Colombia, Ecuador, French Guiana, Guyana, Peru. Surinam, Venezuela

\section{Paleosuchus trigonatus}

Schneider's Smooth-fronted Caiman

Bolivia, Brazil, Colombia, Ecuador, French Guiana, Guyana, Penu, Surinam, Venezuela 


\section{Family: CROCODYLIDAE}

\section{Crocodylus acutus}

American Crocodile

Belize, Colombia, Costa Rica, Ecuador, Greater Antilles (Cuba, Domonican Republic, Haiti, Jamaica), Guatemala, Honduras, Mexico, Nicaragua, Panama, Peru, El Salvador, USA (Florida), Venezuela

\section{Crocodylus cataphractus}

African Shano-nosed or Slender-snouted Crocodile

Angola (Cabinda), Benin, Cameroon, Central African Republic, Chad, Congo, Equatorial Guinea, Gabon, Gambia, Ghana,

Guinea, Guinea Bissau? Ivory Coast, Liberia, Mali, Mauritania, Nigeria, Senegal, Sierra Leone, Tanzania (Ujiji), Togo, Upper Volta, Zaire, Zambia

\section{Crocodylus intermedius}

Orinoco Crocodile

Colombia, Venezuela, (and single record from Grenada)

\section{Crocodylus johnsoni (C.johrstoni)}

Australian Fresh-water Crocodile, Johnson's Crocodile

Australia

\section{Crocodylus moreletii}

Morelet's Crocodile

Belize, Guatemala, Mexico

\section{Crocodylus niloticus}

Nile Crocodile

Angola, Benin, Botswana, Burundi, Cameroon, Central African Republic, Chad, Congo, Egypt, Equatorial Guinea, Ethiopia, Gabon, Gambia, Ghana, Guinea, Guinea Bissau, Ivory Coast Kenya, Liberia, Madasgascar, Mali, Malawi, Mozambique, Namibia, Niger, Nigeria, Rwanda, Senegal, Sierra Leone, Somalia, South Africa, Sudan, Swaziland, Tanzania, Togo, Uganda, Upper Volta, Zaire, Zambia, Zimbabwe

Crocodylus novaeguineae sub-spp. C.n.mindorensis

Mindoro or Philippines Crocodile

Philippine Islands

(sometimes treated as a full species, C. mindorensis, as in RDB)

\section{C.n.novaeguineae}

New Guinea Crocodile

Indonesia (Āru Islands, West Irian), Papua New Guinea

\section{Crocodylus palustris}

Mugger, Marsh Crocodile, Broad-snouted Crocodile

Bangladesh, India, Iran (Sarbaz River), Nepal, Pakistan, Sri Lanka,

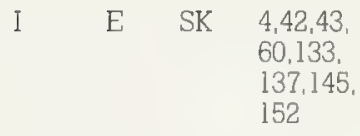

I E SK 4,42,43

60,133 .

137,145

152

(Burma?) 

from the Cocos-Keeling Islands and Fiji Islands. Extinct in Singapore

\section{Crocodylus rhombifer}

Cuban Crocodile

Cuba (inc. Isla de Pinos)

\section{Crocodylus siamensis}

Siamese Crocodile

Indonesia (Bomeo, Java), Kampuchea, Laos, Malaysia (peninsula), Thailand, Vietnam

\section{Osteolaemus tetraspis}

West African Dwarf Crocodile

Angola (Cabinda), Benin, Cameroon, Central African Republic, Congo, Gabon, Gambia, Ghana, Guinea, Guinea Bissau, Ivory Coast, Liberia, Nigenia, Senegal, Sienra Leone, Togo, Uganda, Upper Volta, Zaire

(the Zaire population is sometmes treated as a full species, O.osbomi)

\section{Tomistoma schlegelii}

False Gharial, False Gavial

Indonesia (Borneo, Sumatra), Malaysia (peninsula, Sarawak),

Thailand (possibly extinct)

\section{Family: GAVIALIDAE}

\section{Gavialis gangeticus}

Gharial, Gavial

Bangladesh, Bhutan (probably extinct), India, Nepal, Pakistan

Crocodylus porosus
Estuarine or Salt-water Crocodile
Australia, Bangladesh, Brunei, Burma, India (inc. Andaman
Islands), Indonesia, Kampuchea, Malaysia, Palau Islands, Papua
New Guinea, Philippine Islands, Solomon Islands, Sri Lanka,
Thailand, Vanuatu, Vietnam, also records of wandering individuals

Crocodylus porosus
Estuarine or Salt-water Crocodile
Australia, Bangladesh, Brunei, Burma, India (inc. Andaman
Islands), Indonesia, Kampuchea, Malaysia, Palau Islands, Papua
New Guinea, Philippine Islands, Solomon Islands, Sri Lanka,
Thailand, Vanuatu, Vietnam, also records of wandering individuals

Crocodylus porosus
Estuarine or Salt-water Crocodile
Australia, Bangladesh, Brunei, Burma, India (inc. Andaman
Islands), Indonesia, Kampuchea, Malaysia, Palau Islands, Papua
New Guinea, Philippine Islands, Solomon Islands, Sri Lanka,
Thailand, Vanuatu, Vietnam, also records of wandering individuals

Crocodylus porosus
Estuarine or Salt-water Crocodile
Australia, Bangladesh, Brunei, Burma, India (inc. Andaman
Islands), Indonesia, Kampuchea, Malaysia, Palau Islands, Papua
New Guinea, Philippine Islands, Solomon Islands, Sri Lanka,
Thailand, Vanuatu, Vietnam, also records of wandering individuals

Crocodylus porosus
Estuarine or Salt-water Crocodile
Australia, Bangladesh, Brunei, Burma, India (inc. Andaman
Islands), Indonesia, Kampuchea, Malaysia, Palau Islands, Papua
New Guinea, Philippine Islands, Solomon Islands, Sri Lanka,
Thailand, Vanuatu, Vietnam, also records of wandering individuals

Crocodylus porosus
Estuarine or Salt-water Crocodile
Australia, Bangladesh, Brunei, Burma, India (inc. Andaman
Islands), Indonesia, Kampuchea, Malaysia, Palau Islands, Papua
New Guinea, Philippine Islands, Solomon Islands, Sri Lanka,
Thailand, Vanuatu, Vietnam, also records of wandering individuals

$\begin{array}{lll}\text { I E SK } & 42,43,44 . \\ \text { II } & 85,98,130 \\ \text { (Papua New } & & 147,149 . \\ \text { Guinea) } & & 152\end{array}$

\section{Guinea)}

E SK $42,43,60$,

145,152

$42,43,98$ 152

$\begin{array}{lll}\text { E SK } & \begin{array}{l}42,43,98 \\ 152\end{array}\end{array}$

I Id $\begin{array}{ll}\text { SK } & 42,43,93 \\ \text { (F) } & 152\end{array}$

I $\quad$ E SK $\quad 42,43,152$

\section{Order: RHYNCHOCEPHALIA}

Family: SPHENODONTIDAE

\section{Sphenodon punctatus}

Tuatara

New Zealand 
Order: SQUAMATA,

Lizards, Snakes, Amphisbaenians

Suborder: SAURIA, Lizards

\section{Family: GEKKONIDAE}

\section{Cyrtodactylus serpensinsula}

(Gymnodactylus serpensinsula)

Serpent Island Gecko

Round Island, Serpent Island, (Mauritius)

\section{Oedura reticulata}

II $\quad \mathrm{R}-12,43$

Reticulated Velvet Gecko

Australia

\section{Phelsuma spp.}

Genus Phelsuma: Day Geckos, certain species may appear in the Live

Animal trade. The systematics of this genus is subject to some dispute.

\section{Phelsuma abbotti}

Abbott's Day Gecko

Aldabra, Amirante Islands, Assumption, Cosmoledo Island,

Madagascar, Seychelle Islands

(P.a. longinsulae and P.a pulchra from the Seychelles are sometmes regarded as a separate species, P.longinsulae)

\section{Phelsuma agalegae}

Agalega Island (Mauritius)

\section{Phelsuma andamanensis}

Andaman Day Gecko

Andaman Islands (India)

\section{Phelsuma astriata}

Astove Island, Seychelle Islands

Phelsuma barbouri

Madagascar

\section{Phelsuma bimaculata}

Madagascar

(sometunes treated as a subspecies of P.quadriocellata)

\section{Phelsuma breviceps}

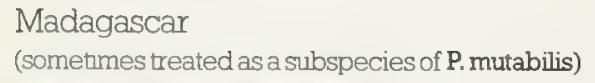


Phelsuma comorensis

II

Comoro Islands

(sometmes treated as a subspecies of P. dubia)

\section{Phelsuma dubia}

Madagascar, Tanzania (inc. Zanzibar)

\section{Phelsuma edwardnewtonii (P. newtoni)}

Rodriguez Day Gecko

Rodriguez Island (Mauritius)

(has been reported extinct, see Ref. 82)

\section{Phelsuma flavigularis}

Madagascar

\section{Phelsuma guentheri}

Round Island Day Gecko

Round Island (Mauritius)

\section{Phelsuma guimbeaui}

Mauritius

\section{Phelsuma guttata}

Madagascar

Phelsuma laticauda

Gold-dust Day Gecko

Comoro Islands, Farquhar Island (Seychelles)?, Madagascar

\section{Phelsuma lineata}

Side-striped Day Gecko

Madagascar

\section{Phelsuma madagascariensis}

Madagascar Day Gecko

Madagascar, Seychelle Islands

(P.m. sundbergi from the Seychelles is frequently regarded as a full species)

Phelsuma modesta

Madagascar

\section{Phelsuma mutabilis}

Madagascar

Phelsuma ocellata (Rhoptropella ocellata)

Namibia, South Africa

Phelsuma ornata (P. vinsoni)

Mauritius (inc. Round Island), Reunion 


\section{Phelsuma parkeri}

Pemba Island (Tanzania)

(sometimes treated as a subspecies of P. abbotti)

\section{Phelsuma quadriocellata}

Madagascar

\section{Phelsuma serraticauda}

Madagascar

(sometimes treated as a subspecies of P. flavigularis)

\section{Phelsuma standingi}

Madagascar

\section{Phelsuma trilineata}

Madagascar

\section{Phelsuma v-nigra}

Comoro Islands

(sometimes treated as a subspecies of P. abbotti)

Family: PYGOPODIDAE

\section{Paradelma orientalis}

Queensland Snake-lizard

Australia

\section{Family: XANTUSIIDAE}

\section{Xantusia riversiana (Klauberina riversiana)}

Island Night Lizard

USA (Califomia)

\section{Family: AGAMIDAE}

\section{Hydrosaunus pustulatus}

Sail-fin Lizard, Soa-Soa Water Lizard, (Crested Lizard)

Philippine Islands

\section{Uromastyxspp.}

Species of Uromastyxare widely termed Spiny-tailed Lizards, Palm Lizards, or Mastigures

\section{Uromastyx acanthinurus}

Black Spiny-tailed Lizard 
Uromastyx aegyptius

Egyptian Spiny-tailed Lizard

Egypt, Iraq, Israel, Saudi Arabia, probably Jordan

Uromastyx asmussi

(Hom Lizard)

Afghanistan, Iran, Pakistan

Uromastyx benti

South Yemen

\section{Uromastyx hardwiclai}

II $\quad$ - F 7.72

Hardwick's Spiny-tailed Lizard

Afghanistan, India, Pakistan

Uromastyx loricatus

Iran, Iraq

Uromastyx macfadyeni

Ethiopia, Somalia

Uromastyx microlepis

(Hom Lizard Small-grain)

Bahrain, Iran, Iraq, Kuwait, Oman, Saudi Arabia, South Yemen

United Arab Emirates, probably also Qatar and North Yemen

Uromastyx ocellatus

Egypt, Sudan

Uromastyx ormatus

Egypt, Israel, Jordan, Saudi Arabia, Syria

\section{Uromastyx philbyi}

Saudi Arabia

Uromastyx princeps

Ethiopia, Somalia

\section{Uromastyx thomasi}

Oman

Family: CHAMAELEONIDAE

Chamaeleo adolfifriderici

Rwanda, Uganda, Zaire 
Chamaeleo affinis

Ethiopia, Somalia, probably Djibouti

Chamaeleo africanus

African Chameleon

Cameroon, Chad, Egypt, Ethiopia, Mali, Niger, Nigeria,

Somalia, Sudan, probably Djibouti and Upper Volta

Chamaeleo anchietae

Angola, Tanzania, Zaire

Chamaeleo angeli

Madagascar

Chamaeleo antimena

Madagascar

(formerly inc. in C. rhinoceratus)

Chamaeleo balteatus

II

Two-banded Chameleon

Madagascar

(sometimes treated as a subspecies of C.bifidus)

Chamaeleo belalandaensis

Madagascar

Chamaeleo bifidus

Madagascar

Chamaeleo bitaeniatus*

Burundi, Ethiopia, Kenya, Rwanda, Somalia, Sudan, Tanzania,

Uganda, Zaire

Chamaeleo boettgeri

Madagascar

Chamaeleo brevicornis

Short-homed Chameleon

Madagascar

Chamaeleo calyptratus

South Yemen, North Yemen

Chamaeleo campani

Madagascar 
Chamaeleo capuroni

Madagascar

Chamaeleo campenteri

Uganda, Zaire

Chamaeleo cephalolepis

Comoro Islands

Chamaeleo chamaeleon

Common or Mediterranean Chameleon

Algeria, Cyprus, Egypt, Gibraltar?, Greece (Crete only), India, Israel, Italy (Sicily only), Jordan, Lebanon, Libya, Morocco, Oman, Pakistan, Portugal, Saudi Arabia, South Yemen, Spair (inc. Canary Islands), Sri Lanka, Syria, Tunisia, Turkey,

North Yemen

(populations in Indıa, Pakistan and Sri Lanka are sometımes treated as a full species, C.zeylanicus, and Arabian populations as C.arabicus

\section{Chamaeleo chapini}

Zaire

\section{Chamaeleo cristatus}

Crested Chameleon

Cameroon, Equatorial Guinea, Gabon, Nigeria

Chamaeleo cucullatus

Madagascar

\section{Chamaeleo deremensis}

Tanzania (Usambara Mts)

\section{Chamaeleo dilepis}

Flap-necked Chameleon

Angola, Botswana, Burundi, Cameroon, Congo, Equatorial Guinea, Ethiopia, Gabon, Kenya, Malawi, Mozambique, Namibia, Nigeria, Rwanda, Somalia, South Africa, Tanzania, (inc. Pemba Island and Zanzibar), Uganda, Zaire, Zambia, Zimbabwe

Chamaeleo eisentrauti

Cameroon

Chamaeleo fallax

Madagascar

Chamaeleo fischeri

Fischer's Chameleon

Kenya, Tanzania

Chamaeleo fuelleborni

Tanzania 
Chamaeleo furcifer

Forked Chameleon

Madagascar

Chamaeleo gallus

Madagascar

Chamaeleo gastrotaenia

Madagascar

Chamaeleo globifer

Madagascar

\section{Chamaeleo goetzei}

Malawi, Tanzania, Zambia

\section{Ghamaeleo gracilis}

Graceful Chameleon

Angola, Benin, Cameroon, Central African Republic, Chad,

Congo, Ethiopia, Equatorial Guinea, Gabon, Gambia, Ghana,

Guinea, Guinea Bissau, Ivory Coast, Kenya, Liberia, Mali, Nigeria,

Senegal, Sierra Leone, Somalia, Sudan, Tanzania, Togo,

Uganda, Zaire, probably Djbouti

Chamaeleo guibei

Madagascar

Chamaeleo hoehnelii

Kenya, Uganda

Chamaeleo incornutus

Tanzania

\section{Chamaeleo jacksonii}

Jackson's Three-homed Chameleon

Kenya, Tanzania

\section{Chamaeleo johnstoni}

Bunundi, Central African Republic, Rwanda, Uganda, Zaire

\section{Chamaeleo labordi}

Madagascar

(sometumes treated as a subspecies of $\mathbf{C}$. rhinoceratus)

\section{Chamaeleo lateralis}

\section{Madagascar}

\section{Chamaeleo laterispinis}


Chamaeleo linotus

Madagascar

Chamaeleo malthe

Madagascar

Chamaeleo marshalli

Short-tailed Dwarf Chameleon

Mozambique, Zimbabwe

(alternatuvely regarded as a member of the Dwarf Chameleon genus Rhampholeon)

Chamaeleo melleri

Meller's Chameleon

Malawi, Tanzania

Chamaeleo minor

Madagascar

Chamaeleo mlanjensis

Malawi

Chamaeleo monachus

Socotra Chameleon

Socotra (South Yemen)

\section{Chamaeleo monoceras}

Madagascar

Chamaeleo montium (inc. C.feae)

Mountain Chameleon

Cameroon, Equatorial Guinea, Nigeria

Chamaeleo namaquensis

Desert Chameleon

Angola, Namibia, South Africa

\section{Chamaeleo nasutus}

Madagascar

Chamaeleo oshaughnessyi

Madagascar

\section{Chamaeleo oustaleti}

Oustalet's Giant Chameleon

Madagascar, (and introduced in Ngong Forest near Nairobi, Kenya)

\section{Chamaeleo oweni}

Owen's Three-homed Chameleon

Cameroon, Congo, Equatorial Guinea, Gabon, Nigeria, Zaire 
Panther Chameleon

Madagascar, (introduced on Reunion)

\section{Chamaeleo parsonii}

Parson's Giant Chameleon

Madagascar

\section{Chamaeleo peyrieresi}

Madagascar

\section{Chamaeleo pfefferi}

Cameroon

\section{Chamaeleo polleni}

Comoro Islands

\section{Chamaeleo pumilis}

Variegated Dwarf Chameleon

Mozambique, Namibia, South Africa

\section{Chamaeleo quadricomis}

Four-homed Chameleon

Cameroon, Nigeria

\section{Chamaeleo quilensis}

Angola, Benin, Botswana, Cameroon, Congo, Equatorial Guinea Gabon, Kenya, Malawi, Mozambique, Namibia, Nigeria, South Africa, Tanzania (including Pemba and Zanzibar), Togo Uganda, Zaire, Zambia, Zimbabwe

\section{Chamaeleo rhinoceratus}

Madagascar

\section{Chamaeleo rudis*}

Burundi, Kenya, Rwanda, Tanzania, Uganda

\section{Chamaeleo senegalensis}

Senegal Chameleon

Benin, Bunundi, Cameroon, Central African Republic, Ethiopia, Gambia, Ghana, Guinea, Guinea Bissau, Ivory Coast, Kenya, Liberia, Mali, Nigeria, Rwanda, Senegal, Sierra Leone, Sudan, Tanzania, Togo, Uganda, Zaire, Zambia

\section{Chamaeleo spinosus}

Spiny Chameleon

Tanzania

\section{Chamaeleo tempeli}

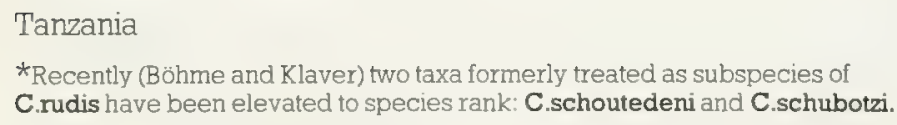


Chamaeleo tenuis

Slender Chameleon

Tanzania

Chamaeleo tigris

Seychelle Islands

Chamaeleo tsaratananensis

Madagascar

Chamaeleo tuzetae

Madagascar

Chamaeleo verrucosus

Madagascar

Chamaeleo werneri

Tanzania

Chamaeleo wiedersheimi

Cameroon, Nigeria

Chamaeleo willsii

Madagascar

Chamaeleo xenorhinus

Uganda, Zaire

Family: IGUANIDAE

Amblyrhynchus cristatus

Galapagos Marine Iguana

Galapagos Islands (Ecuador)

\section{Anolis roosevelti}

Giant Anole, Culebra Giant Ancle

Isla Culebra (Puerto Rico)

(possibly extinct)

Brachylophus fasciatus

Fiji Banded Iguana

Fiji Islands, Tonga Islands

Brachylophus vitiensis

Fiji Crested Iguana

Fiji Islands (Yaduataba Island)

Conolophus pallidus

Barington Land Iguana

Santa Fé (=Barrington) Island in Galapagos group (Ecuador) 


\section{Cyclura carinata}

Turks and Caicos Ground Iguana

Bahama Islands, Turks and Caicos Islands

Cyclura collei (C.lophoma) - possibly extinct

Jamaica's Ground Iguana

Jamaica

\section{Cyclura comuta (inc. C.stejnegeri)}

Rhinoceros Iguana

Dominican Republic, Haiti, Isla Mona, Navassa Id.

(in RDB C.c.stejnegeri from Isla Mona is in category ' $\mathrm{V}$ ', while C.c.comuta from the rest of the range is in Preamble 9-probably threatened)

\section{Cyclura cychlura (inc. C.baelopha, C.figginsi, plus C.inornata)}

Bahama Islands

\section{Cyclura nubila (inc. C.caymanensis, C.macleayi)}

Cayman Islands, Cuba

\section{Cyclura pinguis}

Anegada Ground Iguana

Anegada Id. (British Virgin Islands)

\section{Cyclura ricordi}

Hispaniola Ground Iguana, Ricord's Ground Iguana

Dominican Republic, Haiti

\section{Cyclura rileyi (inc. C.cristata, C.nuchalis)}

\section{Bahama Islands}

(In RDB C.r.nileyi is in category ' $E$ ' the other two races are in ' $R$ ')

\section{Gambelia silus (Crotaphytus silus, C.wislizenii silus)}

Blunt-nosed or San Joaquin Leopard Lizard

USA (Califomia)

\section{Iguana delicatissima}

Lesser Antilles (inc. Anguilla, Antigua, lle Chevreau,

La Désiderade, Dominica, Ile Fourchue, Les Iles Frégates,

Guadeloupe, Martinique, Nevis, Les Iles des Saints,

St. Barthélemy, St. Eustatius, St. Martin)

\section{Iguana iguana}

Common Iguana, Green Iguana, (South American 'Chameleon') Belize, Bolivia, Brazil, Colombia, Costa Rica, Ecuador, French Guiana, Guatemala, Guyana, Honduras, Lesser Antilles (inc. Grenada, the Grenadines, Guadaloupe, Montserrat, Saba, Les Iles des Saints, St. Lucia, St. Vincent, Virgin Islands), Mexico, Netherlands Antilles, Nicaragua, Panama, Paraguay, Peru, El Salvador, Surinam, Trinidad \& Tobago, Venezuela 
Phrynosoma coronatum blainvillei

San Diego Homed Lizard

Mexico (Baja Califomia), USA (Califomia)

\section{Sauromalus varius}

San Esteban Island Chuckwalla

Mexico (San Esteban Island)

Family: LACERTIDAE

Podarcis filfolensis filfolensis (Lacerta f.filfolensis)

Filfola Lizard

Filfola Island (Malta)

Gallotia simonyi (Lacerta simonyi)

Hierro Giant Lizard

Canary Islands (Hierro Island)

Family: CORDYLIDAE

\section{Cordylus campbelli}

Namibia

\section{Cordylus cataphractus}

South Africa

\section{Cordylus coerulopunctatus}

South Africa

\section{Cordylus cordylus}

Āngola, Botswana, Ethiopia, Kenya, Lesotho?, Malawi,

Mozambique, Namibia, South Africa, Swaziland?

Tanzania, Zaire, Zambia, Zimbabwe

\section{Cordylus giganteus}

Lesotho, South Africa

\section{Cordylus macropholis}

South Africa

\section{Cordylus namaquensis}

Namibia

\section{Cordylus peersi}

South Africa

\section{Cordylus polyzonus}

Botswana, Lesotho?, Namibia, South Africa

\section{Cordylus ukingensis}




\section{Cordylus vittifer}

II

Angola, Botswana, Lesotho?, Namibia?, South Africa,

Swaziland

\section{Cordylus warreni}

Lesotho? Mozambique, South Africa, Swaziland?,

Zimbabwe

Pseudocordylus capensis

South Africa

Pseudocordylus langi

Lesotho, South Africa

Pseudocordylus microlepidotus

Namibia, South Africa

Pseudocordylus robertsi

II

South Africa

Pseudocordylus spinosus

II

South Africa

Family: TEIIDAE

\section{Ameiva polops}

St. Croix Ground Lizard

St. Croix and adjacent cays (US Virgin Islands)

\section{Cnemidophorus hyperythrus}

Orange-throated Whiptail

Mexico (Baja Califomia), USA (Califomia)

\section{Crocodilurus lacertinus}

Brazil, French Guiana, Guyana, Surinam

\section{Dracaena guianensis}

Caiman Lizard, (Croco-Teju)

Brazil, French Guiana, Peru, possibly Guyana and other countries bordering the Amazon basin

Tupinambis rufescens (inc. T.duseni and

T.teguixin sebastiani)

(Argentine Teju, Red Teju)

Argentina, Bolivia, Brazil, Paraguay, Unuguay

Tupinambis teguixin (Argentine or Brazil Teju)

(inc. T.nigropunctatus)(Jacarura),

Il

Common Tegu or Banded Tegu

Argentina, Bolivia, Brazil, Colombia, French Guiana, Guyana,

Paraguay, Peru, Surinam, Trinidad \& Tobago, Venezuela, probably Ecuador

40 
Family: SCINCIDAE

Gongylomorphus bojerii fontenayi

R

Macabé Forest Skink

Mauntius

\section{Leiolopisma telfairii}

Round Island Skink

Round Island (Mauritius)

Macroscincus coctaei - probably extinct

Cape Verde Giant Skink

Cape Verde Islands

Family: ANGUIDAE

\section{Gerrhonotus panamintinus}

Panamint Alligator Lizard

USA (California)

\section{Family: ANNIELLIDAE}

\section{Anniella pulchra nigra}

Black Legless Lizard

USA (Califomia)

Family: HELODERMATIDAE

\section{Heloderma horridum}

Beaded Lizard

Guatemala, Mexico

\section{Heloderma suspectum}

Gila Monster

Mexico, USA

\section{Family: VARANIDAE}

\section{Varanus acanthurus}

Spiny-tailed Pygmy Monitor

Australia

Varanus bengalensis (V.monitor, inc. $\mathbf{V}$.nebulosus)

Indian Monitor, Common Monitor (India)

(Agra, Bengal, Indian Small-grain, Penang, Rice,

Talagoya ${ }_{1}$ - Lizard)

Afghanistan, Bangladesh, Burma, India, Indonesia, Iran,

Malaysia, Nepal, Pakistan, Sri Lanka, Thailand, Vietnam, probably Kampuchea and Laos

\section{Varanus brevicauda}

Short-tailed Pygmy Monitor

Australia

Streak-tailed Monitor

Australia 


\section{Varanus dumerilii}

Duméril's Monitor.

II

SK

(Fish Lizard)

Burma, Indonesia, Malaysia, Thailand

\section{Varanus eremius}

Desert Pygmy Monitor

Australia

\section{Varanus exanthematicus}

African Savanna Monitor (African Large-grain Lizard)

Angola, Benin, Botswana, Bunundi, Cameroon, Central African Republic, Chad, Djibouti, Ethiopia, Gambia, Ghana, Guinea, Guinea Bissau, Ivory Coast, Kenya, Lesotho, Malawi, Mali, Mauritania, Mozambique, Namibia, Niger, Nigeria, Rwanda, Senegal, Sierra Leone, Somalia, South Africa, Sudan, Swaziland, Tanzania, Togo, Uganda, Upper Volta, Zaire, Zambia, Zimbabwe

\section{Varanus flavescens}

Yellow Monitor (Calcutta or Indian Oval-grain Lizard) Bangladesh, India, Nepal, Pakistan

\section{Varanus giganteus}

Perentie

Australia

\section{Varanus gilleni}

Pygmy Mulga Monitor

\section{Australia}

\section{Varanus glauerti}

Long-tailed Rock Monitor

Australia

\section{Varanus glebopalma}

Australia

\section{Varanus gouldii}

Sand Monitor, Gould's Monitor

Australia, Indonesia (West Inian), Papua New Guinea

\section{Varanus grayi}

Gray's Monitor

Philippine Islands (Luzon)

\section{Varanus griseus}

Desert Monitor, Agra Monitor (India)

(Agra, Bengal, - Lizard, Baghdad Small-grain Lizard)

Afghanistan, Ālgeria, Chad, Egypt, India, Iran, Iraq, Israel, Jordan, Kuwait, Lebanon, Libya, Mauritania, Morocco, Oman, Pakistan, Qatar, Saudi Arabia, South Yemen, Sudan, Syria, Tunisia, Turkey, United Arab Emirates, USSR, Westem Sahara, North Yemen (the RDB citation applies only to V.g.caspius, the Central Asian or Transcaspian Desert (or Grey) Monitor, from Afghanistan, Iran, Pakistan (northern Baluchistan), and USSR)

\section{Varanus indicus}

Pacific Monitor, Mangrove Monitor, (Ambon or Flower Lizard) 


\section{Varanus karlschmidti}

Sepik Monitor

Papua New Guinea

\section{Varanus Komodoensis}

Komodo Dragon

Indonesia (Flores, Gili Mota, Komodo, Owadi Sami, Padar, Rintja)

\section{Varanus mertensi (in. V.bulliwallah)}

Merten's Water Monitor

Australia

\section{Varanus mitchelli}

Mitchell's Water Monitor

Australia

\section{Varanus niloticus}

Nile Monitor, (African Small-grain Lizard)

Angola, Benin, Botswana, Cameroon, Central African Republic

Chad, Congo, Djibouti, Egypt, Ethiopia, Equatorial Guinea,

Gabon, Gambia, Ghana, Guinea, Guinea Bissau, Ivory Coast,

Kenya, Lesotho, Liberia, Malawi, Mali, Maunitania,

Mozambique, Namibia, Niger, Nigeria, Senegal, Sierra Leone,

Somalia, South Africa, Sudan, Swaziland, Tanzania, Togo,

Uganda, Upper Volta, Zaire, Zambia, Zimbabwe

\section{Varanus prasinus}

Emerald Monitor

Australia (Torres Straits Islands), Indonesia (West Irian),

Papua New Guinea

\section{Varanus primordius}

Ridge-tailed Monitor

Australia

(frequently treated as a subspecies of V.acanthurus)

\section{Varanus rudicollis}

Rough-necked Monitor

(Tree Lizard)

Burma, Indonesia, Malaysia, Thailand

\section{Varanus salvadorii}

Papuan Monitor, Tree Crocodile

Indonesia (West Irian), Papua New Guinea

Varanus salvator (in. V.bivittatus, V.togianus)

Malayan Monitor, Water Monitor

(very many names, in leather trade according to patterning and place of origin, eg., No-mark, Plain, Rice, Ring, Borneo, Java, Philippines, Sumatra, Semi-Java, Singapore, Siam, Ramgodie, Cabragoya - Lizard)

Bangladesh, Burma, China, India (inc. Andaman Is.), Indonesia, Kampuchea, Laos, Malaysia, Philippine Islands, Singapore? Sri Lanka, Thailand, Vietnam

\section{Varanus semiremex}

Mangrove Monitor, Rusty Monitor

Australia

Varanus spenceri

Spencer's Monitor 


\section{Varanus storri}

Dwarf Monitor

Australia

\section{Varanus timorensis}

Timor Tree Monitor, Spotted Tree Monitor

Australia, Indonesia, Papua New Guinea

\section{Varanus tristis}

Freckled Monitor, Moumful Tree Monitor

Australia

Varanus varius

Lace Monitor

Australia

\section{Suborder: SERPENTES, Snakes}

\section{Family: TYPHLOPIDAE}

\section{Typhlops monensis}

Mona Blind Snake

Isla Mona (Puerto Rico)

\section{Family: BOIDAE}

A few taxa of the family Boidae are listed on CITES Appendix I, the remainder are on Appendix II. The actual content of this family has been the subject of recent discussion among herpetologists. Both McDowell (1975, see Ref list B) and Underwood (1976) remove the genera Bolyeria, Casarea, Exiliboa, Trachyboa, Tropidophis and Ungaliophis from the family Boidae. McDowell also removes Loxocemus, while Underwood retains Loxocemus and adds Xenopeltis. Pending discussion, the contents of the Boidae given here closely follows the more conservative list of Stimson (1969), this also appears to be the usage of CITES and RDB. Some changes are made at low taxonomic levels, following Sheplan \& Schwartz (1974), McDowell (1975. 1979), and Collins et al (1978). Python oenpelliensis was newly described by Gow (1977), P.saxuloides by Miller \& Smith (1980), P.bredli by Gow (1981) and P.carinatus by Smith (1981).

\section{Acrantophis dumerili}

Duméril's Boa

Madagascar

\section{Acrantophis madagascariensis}

Madagascar Boa

Madagascar

\section{Aspidites melancophalus}

Black-headed Python

Australia

\section{Aspidites ramsayi}

Woma

Australia
II

II

II

II 


\section{Boa constrictor (Constrictor constrictor)}

Boa Constrictor,

(Ampalague, Giboya, Masacuate)

Argentina, Belize, Bolivia, Brazil, Colombia, Costa Rica, Ecuador, French Guiana, Guatemala, Guyana, Honduras, Lesser Antilles (Dominica, Santa Lucia), Mexico, Nicaragua, Panama, Paraguay, Peru, El Salvador, Suninam, Trinidad \& Tobago, Venezuela

\section{Bolyeria multocarinata}

Round Island Boa

Round Island (Mauritius)

\section{Calabaria reinhardtii}

Calabar Ground Python

Benin, Cameroon, Congo, Equatorial Guinea, Gabon, Ghana,

Ivory Coast, Liberia, Nigeria, Togo, Zaire, probably

Central African Republic

\section{Candoia aspera (Enygrus asper)}

Papuan Ground Boa

Indonesia (Moluccas, West Irian), Papua New Guinea

(inc. Bismarck Archipelago as far east as Bougainville)

\section{Candoia bibroni (Enygrus bibroni)}

Pacific Boa

Fiji Islands (inc. Rotuma), Loyalty Islands, Vanuatu (= New Hebrides), (inc, Banks Islands), Westem Samoa, also Ta'u Island in American Samoa, Solomon Islands (incl. Santa Cruz)

\section{Candoia carinata (Enygrus carinata)}

Solomons Ground Boa

Indonesia (eastem islands inc. Moluccas, Sulawesi, West Irian),

Palau Islands, Papua New Guinea (inc. Bismarck Archipelago),

Solomon Islands, (incl. Santa Cruz)

\section{Casarea dussumieri}

Round Island Keel-scaled Boa

Round Island (Mauritius)

\section{Charina bottae}

Rubber Boa

Canada, USA

(RDB entry is for Charina b. umbratica, the Southern Rubber Boa,

from isolated localities in Calıfomia)

\section{Chondropython viridis}

Green Tree Python

Australia (Cape York Peninsula), Indonesia (West Irian),

Papua New Guinea (mainland, not Bismarck Archipelago),

Solomon Islands?

\section{Corallus annulatus (Boa annulata)}

Annulated Tree Boa

Colombia, Costa Rica, Ecuador, Nicaragua, Panama

\section{Corallus caninus (Boa canina)}


Corallus enydris (C.hortulanus, Boa hortulanus, inc. Corallus or Boa coola)

Garden Tree Boa

Bolivia, Brazil, Colombia, Costa Rica, Equador, French Guiana, Guyana, Nicaragua, Peru, Surinam, Trinidad \& Tobago, Venezuela, Windward Islands (Grenada, Grenadines, St. Vincent)

\section{Epicrates angulifer}

Cuban Tree Boa

(Maja (de Santa Maria)), Cuba (inc. Isla de Pinos)

RDB entry is for E.a.angulifer from the Isle of Pines and two small islands off the coast of Cuba]

\section{Epicrates cenchria}

Rainbow Boa

(Araramboia)

Argentina, Bolivia, Brazil, Colombia, Costa Rica, Ecuador French Guiana, Guyana, Panama, Paraguay, Peru, Sunnam, Trinidad \& Tobago, Venezuela

\section{Epicrates chrysogaster (E.striatus chrysogaster)}

Bahama Islands Boa

Bahama Islands, Caicos Islands, possibly Turks Islands

\section{Epicrates exsul}

Abaco Island Boa

Bahama Islands

\section{Epicrates fordii (E.inornatus fordii)}

Ford's Boa

Dominican Republic, Haiti

(see note under Epicrates inomatus)

\section{Epicrates gracilis}

Haitian Vine Boa

Dominican Republic, Haiti

\section{Epicrates inomatus}

Puerto Rican Boa

Puerto Rico

(E.inomatus, as used by CITES and RDB, appears to be in the sense of Stull (1935) and Stimson (1969, Das Tierreich 89). In this usage three subspecies are recognised, 1, E i.inomatus (Puerto Rico), 2. E. ifordii (Dominican Republic and Haiti), 3. E.i.granti (St. Thomas and Tortola in the Vurgin Islands) Good evidence has subsequently been proposed (Sheplan \& Schwartz, 1974 followed by Schwartz \& Thomas, 1976) that E.i.fordii is best regarded as a distinct species (E.fordii) and that E.i.granti has greater affinties with the Epicrates of Isla Mona, the latter two taxa are accordingly treated as a distinct species (E.monensis) in the works cited. The present list includes the West Indian species of Epicrates as recognised by Sheplan \& Schwartz (1974) and Schwartz \& Thomas (1976); the proper treatment of E.fordii and E.monensis by CITES and RDB thus remains to be determined, it is assumed that the current CITES and RDB treatment will contmue to apply to Epicrates inomatus (of Sheplan \& Schwartz)

Epicrates monensis (E.gracilis monensis plus E.inomatus granti)

Isla Mona (Puerto Rico), St. Thomas (US Virgin Islands), Tortola (British Virgin Islands) 


\section{Epicrates striatus}

Fischer's Tree Boa

Bahama Islands, Dominican Republic, Haiti

(RDB entry is for E.s.fosteri from the Bahamas)

Epicrates subflavus

Jamaica Boa

Jamaica

\section{Eryx colubrinus}

Kenya Sand Boa

Egypt, Ethiopia, Kenya, Niger, Somalia, Sudan,

Tanzania, North Yemen?

\section{Eryx conicus (Gongylophis conicus)}

Indian Sand Boa

(Baby 'Python')

India, Pakistan, Sri Lanka

\section{Eryx elegans}

Afghanistan, Iran, USSR (Turkmenistan)

\section{Eryx jaculus}

Javelin) Sand Boa, Spotted Sand Boa

Albania, Algeria, Bulgaria, Egypt, Greece, Iran, Iraq, Israel, Jordan, Lebanon, Morocco, Romania (possibly extinct),

Saudi Arabia, Syria, Tunisia, Turkey, USSR, Yugoslavia

\section{Eryx jayakari}

Jayakar's Sand Boa

Bahrain, Kuwait, Oman, Qatar, Saudi Arabia, South Yemen, United Arab Emirates, North Yemen

\section{Eryx johnii}

Blunt-tailed Sand Boa

Afghanistan, India, Iran, Pakistan

\section{Eryx miliaris}

Iran, USSR

\section{Eryx muelleri}

Müller's Sand Boa

Cameroon, Chad, Ghana, Ivory Coast, Mali, Mauritania, Nigeria, Senegal, Sudan, Togo, Upper Volta, probably Benin and Central African Republic

\section{Eryx somalicus}

Somalia

\section{Eryx tataricus}


Brazil

\section{Eunectes murinus}

Anaconda

Bolivia, Brazil, Colombia, Ecuador, French Guiana, Guyana,

Peru, Surinam, Trinidad, Venezuela

\section{Eunectes notaeus}

Yellow Anaconda

Argentina, Bolivia, Brazil, Paraguay, Uruguay

\section{Exiliboa placata}

\section{Mexico}

\section{Liasis albertisii (L.fuscus albertisii)}

D'albertis' Python

Indonesia (West Irian), Papua New Guinea, Torres Straits Islands (Australia)

\section{Liasis boa (Bothrochilus boa, Nardoana boa)}

Barred Python

Papua New Guinea (Bismarck Archipelago, mainland

New Guinea?), Tokelau Islands?

\section{Liasis childreni}

Children's (Rock) Python

Australia

\section{Liasis mackloti (inc. L.fuscus)}

Water Python (common name for L.fuscus)

Australia, Indonesia (Lesser Sunda Islands), Papua New Guinea

\section{Liasis maximus}

(known only from the type specimen, from New Guinea; a possible synonym of L.papuanus (McDowell, 1975 Ref, list B)

\section{Liasis olivaceus}

Olive (Rock) Python

Australia

\section{Liasis papuanus (L.olivaceus papuanus)}

Papuan Python

Indonesia (West Irian), Papua New Guinea

\section{Liasis perthensis}

West Australian Rock Python

Australia

(taxonomic status uncertain, (Cogger, 1975, Ref, list B))

\section{Lichanura trivirgata}

Rosy Boa 


\section{Loxocemus bicolor}

Costa Rica, Guatemala, Honduras, Mexico, Nicaragua,

El Salvador

\section{Python amethistinus (Liasis amethistinus)}

Amethystine (Rock) Python

Australia (Cape York Peninsula, Torres Straits Is.), Indonesia (eastern islands, West Irian), Papua New Guinea, Philippines?

\section{Python anchietae}

Angolan Python

Angola, Namibia

Python boeleni (Liasis boeleni, Liasis taronga)

Boelen's Python

Indonesia (West Irian), Papua New Guinea

\section{Python bredli}

Australia (Northem Tenitory)

\section{Python carinatus}

Rough-scaled Carpet Snake

Australia (Westem Australia)

\section{Python curtus}

Short-tailed Python, Blood Python

Brunei, Indonesia (Bangka, Kalimantan, Sumatra), Malaysia, Thailand (in Malay Peninsula)

\section{Python molurus sub-spp.}

Indian Python, Burmese Python, Oriental Rock Python

Java Tree Python, Molurus Python, Indian Python, Bengal or

Saigon Python)

\section{P.m.bivittatus}

Brunei?, Burma, China, Hong Kong, Indonesia, Kampuchea, Laos, Malaysia, Thailand, Singapore, Vietnam

\section{P.m.molurus}

Bangladesh, India, Nepal, Pakistan, Sri Lanka

\section{Python oenpelliensis}

Australia

\section{Python regius}

Ball Python, Royal Python

Benin, Cameroon, Chad, Gambia, Ghana, Guinea, Guinea Bissau, Ivory Coast, Mali, Nigeria, Senegal, Sierra Leone, Sudan, Togo, Uganda, Upper Volta, Zaire, probably also Central African Republic, Congo, Equatoriảl Guinea, Gabon, Liberia 


\section{Python reticulatus}

Reticulated Python, (Diamond or Java Rock Python)

II - LA

Bangladesh, Brunei, Burma, Indonesia (not to West Inian),

Kampuchea, Laos, Malaysia, Nicobar Islands (India), Philippines

Singapore, Thailand, Vietnam

\section{Python saxuloides}

Lesser African Rock Python

Kenya, probably Malawi, South Africa, possibly Ethiopia,

Somalia, Uganda

\section{Python sebae}

African Python, Water Python, African Rock Python

II $\quad-\quad \underset{\mathrm{SK}}{\mathrm{LA}} 47$

(West African Python)

Angola, Benin, Botswana, Burundi, Cameroon, Central African Republic, Chad, Congo, Djibouti, Equatorial Guinea, Ethiopia, Gabon, Gambia, Ghana, Guinea, Guinea Bissau, Ivory Coast, Kenya, Lesotho, Liberia, Malawi, Mali, Maunitania, Mozambique, Namibia, Nigeria, Rwanda, Senegal, Sierra Leone, Somalia, South Africa, Sudan, Swaziland, Tanzania, Togo, Uganda, Upper Volta, Zimbabwe, Zaire, Zambia

Python spilotus (Morelia spilotes, Morelia argus) P.s.variegata-Carpet Python, P.s.spilotus-Diamond Python Australia, Indonesia (West Irian), Papua New Guinea

Python timorensis

Timor Python

Indonesia (Flores, Timor)

Sanzinia madagascariensis

Madagascar Tree Boa

Madagascar

\section{Trachyboa boulengeri}

Colombia, Ecuador, Panama

\section{Trachyboa gularis}

Ecuador

\section{Tropidophis battersbyi}

Ecuador

\section{Tropidophis canus}

Bahama Islands

\section{Tropidophis caymanensis}

Caymans Ground Boa

Cayman Islands

\section{Tropidophis feicki}


Tropidophis greenwayi

Caicos Islands

Tropidophis haetianus

Haitian Ground Boa

Cuba, Dominican Republic, Haiti Jamaica

Tropidophis maculatus

Cuba

Tropidophus melanurus

Cuba, Navassa Island

Tropidophis nigriventris

Cuba

Tropidophis pardalis

Cuba

Tropidophis paucisquamis

Brazil, Peru

Tropidophis pilsbryi

Cuba

Tropidophis semicinctus

Cuba

Tropidophis taczanowskyi

Ecuador, Peru, Brazil?

Tropidophis wrighti

Cuba

Ungaliophis continentalis

Guatemala, Honduras, Mexico

Ungaliophis panamensis

Colombia, Costa Rica, Nicaragua, Panama

Xenoboa cropani

Brazil 


\section{Family: COLUBRIDAE}

\section{Clelia clelia (Pseudoboa cloelia)}

Musurana

Argentina, Belize, Bolivia, Brazil, Colombia, Costa Rica, Ecuador, French Guiana, Guatemala, Guyana, Honduras, Lesser Antilles (inc. Dominica, Grenada, St. Lucia), Nicaragua, Panama, Paraguay, Peru, El Salvador, Surinam, Trinidad, Uruguay, Venezuela

(snakes from Mexico and elsewhere in Central America formerly assigned to this species are now usually regarded as a full species, Clelia scytalina)

\section{Drymarchon corais couperi}

Eastem Indigo Snake

USA

\section{Elachistodon westermanni}

Indian Egg-eating Snake

Bangladesh, India, Nepal

\section{Hydrodynastes gigas (Cyclagras gigas)}

South American Water 'Cobra'

(Beach 'Cobra', Surucucu)

Argentina, Bolivia, Brazil, Paraguay

\section{Masticophis flagellum ruddocki}

San Joaquin Coachwhip

USA (California)

Masticophis lateralis euryxanthus

Almeda Striped Racer

USA (California)

Nerodia fasciata taeniata (Natrix f.taeniata)

Atlantic Saltmarsh Snake

II - LA -

USA (Florida)

Nerodia sipedon insularum (Natrix s.insularum)

Lake Erie Water Snake

Lake Erie (Canada/USA)

Thamnophis couchi gigas (T.elegans gigas)

Giant Garter Snake

USA (California)

Thamnophis couchi hammondi

(T. elegans hammondi)

Two-striped Garter Snake Mexico (Baja Califomia), USA (California)

Thamnophis sirtalis tetrataenia San Francisco Garter Snake II - - $\underset{\text { SK }}{\mathrm{LA}}-$ USA (Califomia) 
Family: ELAPIDAE

\section{Naja oxiana}

(frequently treated as a subspecies of the Astatic Cobra, Naja naja)

Central Asian or Oxus Cobra

Afghanistan, India (Kashmir, Punjab), Iran (northeast), Pakistan, (north and northwest), USSR (Transcaspian region)

\section{Ogmodon vitianus}

Fiji Snake

Fiji (Viti Levu)

Family: VIPERIDAE

\section{Bothrops insularis}

Queimada Island Bothrops

Brazil (Queimada Island)

\section{Crotalus unicolor}

Aruba Island Rattlesnake

Netherlands antilles (Anuba Island)

\section{Crotalus willardi}

Ridge-nosed Rattlesnake

Mexico, USA (Arizona, New Mexico)

(in RDB C.w.obscurus, the New Mexıco Ridge-nose Rattlesnake, is

Endangered, the four other subspecies are Vulnerable)

\section{Vipera ammodytes transcaucasiana}

Transcaucasian Long-nosed Viper

USSR (Georgia), Turkey

\section{Vipera latifii}

Latifi's Viper

Iran (Lar Valley in Elburz Mtns.)

\section{Vipera lebetina schweizeri}

Blunt-nosed Viper, Levantine or Lebetine Viper

(names applied to V.lebetina as a whole)

Greece (Cyclades only: Kimolos, Milos, Polyagos, Siphnos, possibly Antimilos, Kithnos)

\section{Vipera xanthina raddei}

Armenian Viper

USSR, Turkey 


\section{Additions and Amendments}

CITES RDB Exp Ref

Family: KINOSTERNIDAE

Kinosternon flavescens spooneri

- E - 152

Illinois Mud Turtle

USA 


\section{(A) Sources of information on the survival status of particular species or groups of species.}

The number of each Reference corresponds to entries in the "Ref column of the taxonomic list. The utility of the References given is rather variable; some works concentrate entirely on survival status and conservation, while others may only make a brief comment on these topics in the course of a largely taxonomic treatment. Some of the worksare popular or semi-popular, whlle others are more rigorous in approach. Emphasis is given to more recent works, including those not cited in the third edition of the Red Data Book; the latter (item 43 in the list) contains references to many other works. It is not the intention to provide a complete bibliography of the extensive literature treating the biology and conservation of amphibians and reptiles

1 ANON. 1971. Santa Cruz Long-toed Salamander granteda reprieve. Herpetology 5(4):14.

2. ANON. 1974. Pennsylvania protects the Bog Turtle. Herpet. Rev. 5(2):44.

3 ANON. 1976. Madhya Pradesh Gharial Survey. Newsl. Madras Snake Pk. Trust 1(2):2-3.

4 ASHTON, R.E., Chaurman 1973-74 SSAR Liaison Committee to Regional Societies. Endangered and Threatened Amphibians and Reptiles in the United States. Society for the study of Amphibians and Reptles, Herpetological Circular No. 5

5. AUFFENBERG, W. 1979. A monitor lizard in the Philippines. Oryx 15:39-46.

6. BACON, P.R. 1975. Review on research, exploitation and management of the stocks of Sea Turtles. FAO Fisheries Circular No. 334. (Caribbean and western Ätlantic regions)

7 BHATIA, M.L \& TILAK, R. 1976. Some notes on the Spiny-tailed Lizard (Uromastyx hardwickii) Gray Newsl. Madras Snake Pk. Trust l(2):?.

8. BROWN, L. E. 1975. The status of the near extınct Houston Toad (Bufo houstonensis) with recommendations for its Conservation. Herpet. Rev. 6(2):37-40.

9 BROWN, W.S. 1974. Ecology of the Aquatic Box Turtle Terrapene coahuila (Chelonia, Emydidae) in northem Mexico. Bull. Fla. St. Mus. (biol. Sci.) 19(1):1-67.

10 BUDEN, D.W. 1975. Notes on Epicrates chrysogaster (Serpentes: Boidae) of the southern Bahamas, with description of a new subspecies. Herpetologica 31(2):167-177.

11 BULL, P.C. \& WHITTAKER, A.J. 1975. The amphibians, reptles, birds and mammals. In, Kuschel, G. (Ed.) Biography and Ecology in New Zealand. Junk, The Hague. In series Monographiae biol. 27:231-276.

12. BULLOCK, D. 1977. Round Island-a Tale of Destruction. Oryx 14(1):51-58.

13. BURTON, J.A. \& LAMBERT, M. 1977. Tortoises come to Bntain. In, Sitwell, N. (Ed.) The World of Wildlife. Hamlyn, London

14. BURY, R. B. \& RUTH, S. B. 1972. Santa Cruz Long-toed Salamander: survival in doubt. Herpet. Rev. 4(1):20-22.

15. BURY, R. B. \& MARLOW. R.W. 1973. The Desert Tortoise-will it survve? Natn. Pk. Conserv. Mag. 47:9-12.

16. BUSÄCK, S.D. 1976. A review of the biology of the Gold-striped Salamander, Chioglossa lusitanica (Amphibia: Salamandridae), Biol. Conserv. 10:309-319.

17 BUSTARD, H.R. 1970. The present status of Sea Turtles and the Fiji Iguana in the Fiji Islands. Oryx 10:317-322

18. BUSTARD, H.R. 1974. The Green Turtle. In, Wildl. World Conserv. Yb. 1974:80-87.

19. CAREY, M. 1975. Iguanas on the rocks. Anim. Kingd. 78(2):15-20.

20. CAREY, M. 1975. The Rock Iguana, Cyclura pinguis, on Anegada, British Virgin Islands, with notes on Cyclura ricordi and Cyclura cornuta on Hispaniola. Bull. Fla. St. Mus. (biol. Sci.) 19(4):189-234.

21. CAREY M. 1976. Iguanas of the Exumuas. Wildlife (Lond.) 18(2):59-61.

22. CAPR, A. \& STANCYK, S. 1975. Observations on the ecology and survival outlook of the Hawksbill Turtle. Biol. Conserv. 8:161-172

23. CARR, A. 1977. Crisis for the Atlantic Ridley. Letter in, Mrosovsky, N. (Ed.) Marine Turtle Newsl. No. 4:2-3.

24. CHEKE, A.S. 1975. An undescribed Gecko from Agalega: Phelsuma agalegae sp. nov. Bull. Mauritius Inst. 8(1):33-48.

25. COMMITTEE ON RARE AND ENDANGERED AMPHIBIANS AND REPTILES OF MARYLAND. 1973. Endangered Amphibians and Reptles of Maryland: a special report. Bull. Md. Herp. Soc. 9(3):42-100.

26. CROOK, I. \& CROOK, G. 1972. New Zealand's rarest frog. Animals (Lond.) 14(5):I88-190.

27. CROOK, I. 1975. The Tuatara. In, Kuschel, G. (Ed.) Biogeography and Ecology in New Zealand. Junk, The Hague In series Monographiae biol. 27:331-352.

28. DEGENHARDT, W.G. 1973. The Ridge-nosed Rattlesnake: an endangered species. In. Symposium on rare and endangered wildlife of the southwestern United States: 104-113. New Mexico Dept. of Game and Fish, Santa Fe. New Mexico.

29. DESERT TORTOISE PRESERVE COMMITTEE INC. 1975. Desert Tortoise Preserve. Chelonia 2(2):19-20.

30. DIAMOND, AW. 1976. Breeding biology and conservation of Hawksbill Turtles, Eretmochelys imbricata, L. on Cousin Island, Seychelles. Biol. Conserv. 9(3):199-215.

31. ERNST, C.H. \& BURY, R. B, 1977. Clemmys muhlerbergii (Schoepf) Bog Turtle. Catalogue Am. Amphib. Rept. 204.1-204.2.

32. FRANZ, R. \& AUFFENBERG, W. 1974. The Gopher Tortoise in Georgia. Herpet. Rev. 5(3):74-75 (abstr.)

33. FRAZIER, J. 1975. Marine Turtles of the western Indian Ocean. Oryx 13(2):164-175.

34. FREIBERG, M.A. 1974. The Argentune land tortoise, Geochelone chilensis, an endangered species. Bull. Md. Herp. Soc 10(2):39-41. 
35. GANS, C. \& WILLIAMS, E. 1954. Present knowledge of the snake Elachistodon westermanni Reinhardt Breviora No. 36.

36 HARRIS, H.S. \& SIMMONS, R.S. 1975. An endangered species, the New Mexican Ridge-nosed Rattlesnake Bull. Md. Herp. Soc. 11(1):1-7.

37. HIRTH, H.F. 1971. Synopsis of biological data on the Green Turtle Chelonia mydas (Linnaeus) 1758 FAO Fish. Synop. No. 85 (pag. var.).

38. HONEGGER, R.E. 1978. Threatened Amphibians and Reptiles in Europe. European Committee for the Conservation of Nature and Natural Resources. Councll of Europe, Strasbourg. Nature and Environment Series No. 15

39. HUGHES, G.R. 1973. The survival situation of the Hawksbill Sea-Turtle (Eretmochelys imbricata) in Madagascar Biol. Conserv. 5(2):114-118.

40 HUGHES, G.R. 1974. The Sea Turtles of South-East Africa. Investigational Rep. Oceanogr. Res. Inst. Durban. Part I No. 35:1-144, Part II No. 36:1-96.

41. IUCN. 1971. Marine Turtles. IUCN Publ. New. Ser. Suppl. Pap. No. 31 (Proceedings of the 2nd working meeting of Marine Turtle specialists).

42. IUCN. 1973. Crocodiles. IUCN Publ. New Ser. Suppl. Pap. No. 41 (Proceedings of the 2nd working meeting of Crocodile specialists).

43. IUCN. 1979. Red Data Book3, Amphibians and Reptiles, 3rd Ed. Compled by R. E. HONEGGER. Morges Switzerland

44 JONKLASS, R. 1977. Crocodiles in Sri Lanka. Hamadryad (Newsl. Madras Snake Pk. Trust) 2(2):7.

45 JUVIK, J.O. \& BLANC, C.P. 1974. The Angonoka of Cape Sada. Arimals (Lond.) 16(4); 148-153.

46 JUVIK, J.O. 1975. The Radiated Tortoise of Madagascar. Oryx 13(2):145-148.

47. McLACHLAN, G.R. 1978. South African Red Data Book-Reptiles and Amphibians. S. African National Scientific Programmes Report No. 23,

48. McMORRIS, J.R. \& BURNS, D. M. 1975, Notes on Geochelone impressa. Chelonia 2(2):5-7.

49. MEANS, D. B. \& LONGDEN, C.J. 1976. Aspects of the biology and zoogeography of the Pine Barrens Treefrog (Hyla andersonii) in Northern Florida. Herpetologica 32(2):117-130.

50. MENDEZ, E. 1975. Panama's Golden Frog. Wildl. World Conserv. Yb. 1975:92-95.

51. MITCHELL, R.W. \& SMITH, R.E. 1972. Some aspects of the osteology and evolution of the neotenic spring and cave salamanders (Eurycea, Plethodontidae) of Central Texas. Texas J. Sci. 23(3):343-362.

52. MITTERMEIER, R.A. \& WILSON, R.A. 1974. Redescription of Podocnemis erythrocephala (Spix, 1824), an Amazonian Pelomedusid Turtle. Papéis Avvulsos Zool. S. Paulo. 28(8):147-162.

53 MITTERMEIER, R.A. 1975. A Turtle in every pot. Anim. Kingd. 78(2):9-14.

54. MOLL, E. O. 1976. West Malaysian Turtles: utilisation and conservation, Herpet. Rev. 7(4):163-166.

55. NIETSCHMANN, B. 1975. Of Turtles, ambadas, and people. Chelonia 2(6):6-9.

56. OVERMEER, H, 1976. Agrionemys horsfieldi Gray 1844 steppeschildpad. Lacerta 35(2):15-20.

57 OVINGTON, D. 1978. Australian Endangered Species. Mammals, Birds and Reptiles. Cassell Australia Ltd.

58. PHILIBOSIAN, R. \& RUIBAL, R. 1971. Conservation of the lizard Ameiva polops in the Virgin Islands. Herpetologica 27:450-454.

59. PHILIBOSIAN, R. \& YNTEMA, J.A. 1976. Records and status of some Reptiles and Amphibians in the Virgin Islands. I, 1968-1975. Herpetologica 32(1):77-85.

60. POWELL, J. 1971. The status of crocodllians in the United States, Mexico, Central America, and the West Indies. In, IUCN Publ. New Ser. Suppl. Pap. No. 32:72-82.

61. POWELL, J. 1972. The Morelet's Crocodile, Anim. Kingd. 75(1):21-26.

62. PRITCHARD, P.C.H. 1977. Marine Turtles of Micronesia. Chelonia Press, 536 Fufth Ave., San Francisco, CA. 94118, USA

63 RAU, R. 1971. Cape Reserve for one of World's rarest tortoises. Afr. Wildl. 25(3), reprinted Herpet. Rev. 1972.2(1):19.

64. ROBB, J. 1973. Reptiles and Amphibia, In, Williams, G. R. (Ed). The natural history of New Zealand. An ecological survey: 285-303. A. H. \& A.W. Reed, Wellington, Sydney, London.

65. ROBERTS, T. J. 1917. News from Pakistan. Newsl. Madras Snake Pk. Trust. 2(1):5.

66 RUSSELL J. 1974. The largest lizard in the World. In, Wildl. World Conserv. Yo. 1974:88-95.

67. RUTH, S. B. 1974. The current status of the Santa Cruz Long-toed Salamander-an endangered animal Herpet. Rev. 5(1):27-29.

68. SCHULZ, J.P. 1975. Sea Turtles nesting in Surnam, Zool. Verh. Leiden, No. 143.

69. SCHWARZ, F.J. 1977. Reptlia; Testudines; Cheloniidae In, Cooper, J.E, Robinson, S. S. \& Funderberg, J.B (Eds.) Endangered and Threatened Plants and Animals of North Carolina: 303-308. N. C. State Museum of Natural History, Raleigh, N.C

70) SCRIVEN, K. 1976. Leathery Turtles. In, Wildl. World Conserv. Yo. 1976:94-99.

I1 SHAHI, S. P. 1975, Save the Gharial. Wildlife (Lond) 17(12):554-555.

72. SHARMA, I.K. 1977. De Indische doornstaarthagedis, Uromastyx hardwickii. Lacerta 35(4):53-57.

73. SHAW. J.W. 1972. To save a salamander. Anim. Kingd. 75(6):25-27.

74. SMITH, N.J.H. 1975. Destructive exploitation of the South American River Turtle Chelonia 2(5):3-9, 30

55. STEWART, G.R. 1971. Rare, endangered and depleted Āmphibians and Reptiles in Califorma. Herpetology 5(2):29-35. 
76. TEMPLE, S. A. 1974. Last chance to save Round Island. Wildlife (Lond.) 16(8):370-374

77. TEMPLE, S. A 1974. Wildlife in Mauritius to day. Oryx. 12:584-590.

78. TEMPLE, S. A 1977. Castaway reptiles of the Indian Ocean. Anim. Kingd. 80(4):19-27.

79. THORNTON, O.W. \& THOMAS, R.A. 1976. The status of the Houston Toad, Bufo houstonensis. Herpet. Rev. 7(2):98-99. (abstr)

80. ALVAREZ DEL TORO, M. 1975. Morelet's Crocodile. Wildl. World Conserv. Yb. 1975:88-91

8! USSR, MINISTRY OF AGRICULTURE, 1978. Red Data Book of USSR. Lesnaya Promyshlennost. Moscow

82 VINSON, J. \& VINSON, J-M. 1969. The saurian fauna of the Mascarene Islands. Bull. Mauritius Inst. 6(4):203-320.

83. VINSON, J-M. 1975. Notes on the reptiles of Round Island. Bull. Mauritius Inst. 8(1):49-67.

84. WHITAKER, R. 1975. Status and conservation of the Gharial (Gavialis gangeticus). Henpet. Rev. 6(1):2-3.

85. WHITAKER, R. \& WHITAKKER, Z 1978. Sri Lanka Crocodile Survey. Hamadryad (Newsi. Madras Snake Pk. Trust) 3(1):3

86. WILSON, R.J. 1978. Notes on the crocodile Crocodylus niloticus, in Darfur, Westem Sudan. E. Afr. Wildl. J. $16(1): 65-67$

87. ZWINENBERG, A. J. 1974. The leatherback (Dermochelys conacea), one of the largest living reptiles Bull. Md. Herp. Soc. 10(2):42-48.

88. ZWINENBERG, A]. 1975. The Green Turtle (Chelonia mydas), one of the reptiles most consumed by man, needs immediate protection. Bull. Md. Herp. Soc. 1(2):45-63.

89. ZWINENBERG, A. J. 1975. The western swamp tortoise (Pseudemydura umbrina), one of Australia's rarest reptles. Bull. Md. Herp. Soc. 11(1):14-20.

90. ZWINENBERG, A.]. 1976. The Olive Ridley, Lepidochelys olivacea (Eschscholtz, 1829); probably the most numerous marine turtle today. Bull Md. Herp. Soc. 12(3):75-95.

91. ZWINENBERG, A.J. 1977. Kemp's Ridley, Lepidochelys kempii (Gaman, 1880), undoubtedly the mostendangered marine turtle today (with notes on the current status of Lepidochelys olivacea). Bull. Md. Herp. Soc. 13(3):170-192.

92 ZWINENBERG, A.J. 1977. De exploitatie van scheenplaatschildpadden Podocnemis Pelomedusidae. Lacerta 36(1):12-16.

93. ABERCROMBIE, C.L. 1978. Notes on West African Crocodilians (Reptilia, Crocodilia). J.Herpetol.12(2):260-262.

94 ABERCROMBIE, C.L., DAVIDSON, D., HOPE, C.A. \& SCOTT, D.E. 1980. Status of Morelet's Crocodile Crocodylus moreleti, in Belize. Biol.Conserv.17(2):103-113.

95 ALVAREZ DEL TORO, M., MITTERMEIER, R.A., \& IVERSON, J.B. 1979. River Turtle in Danger. Oryx 15(2):170-173.

96. ANDERSON, S.C. 1979. Synopsis of the Turtles, Crocodiles, and Amphisbaenians of Iran. Proc.Calif.Acad.Sci.(Ser.4)61(22):501-528

97. ANDREN, C. \& NILSON, G. 1979. Vipera latifii (Reptilia, Serpentes, Viperidae) an Endangered viper from Lar Valley, Iran, and Remarks on the Sympatric Herpetofauna. J.Herpetol.13(3):335-341.

98. ANON. 1975. The breeding of Crocodiles in captivity at Samut Prakan, Thailand. Tigerpaper(FAO) 2(4):14-16

99. ANON. 1978. Saving the edible turtle (Cyprus). To the point 7(47):46 (news mag. publ. Sandton, RSA)

100. ANON. 1979. Alligator import/export controls adopted. Endangered Species Tech. Bull. (US Fish \& Wildl.) $4(10): 1,4-5$.

101. ANON. 1979. Bolson Tortoise listed as Endangered. Endangered Species Tech. Bull. (US. Fish \& Wildl.) 4(5):6.

102. ANON. 1980. Chinese Alligator. Tigerpaper (FAO) 7(2):28.

103. ARGANO, R. 1978. Project 1474. Marine Turtles-Westand Central Mediterranean. WWF Yearbook 1977-1978:154.

104. AUFFENBERG, W. 1969. Land of the Chaco Tortoise. Internat. Turtle \& Tortoise Soc.).3(3):16-1S, 36-37.

105. AUSTRALIAN NATIONAL PARKS \& WILDLIFE SERVICE. 1977. Information sheets on Australian Endangered Species. Reptiles, No. I. Westem Swamp Turtle (Pseudemydura umbrina).

106. BELL, B.D. 1977. Research uncovers more facts about rare native frogs. Forest and Bird 204:12-17.

107. BELL, B.D. 1978. Observations on the ecology and reproduction of the New Zealand Leiopelmid Frogs Herpetologica 34(4):340-354.

108. BISCHOFF, W., et al. 1979. Ergebnisse einer herpetologischen Exkursion nach Hierro, Kanarische Inseln. Salamandra 15(3):158-175.

109. BLAKE, D.K, \& LOVERIDGE, J.P. 1975. The role of commercial crocodile farming in crocodile conservation Biol. Conserv. 8:261-272.

110 BLASCO, M. 1979. Chamaeleo chamaeleon in the province of Malaga, Spain. Brit.J.Herpetol. 5:839-841.

11 1. BLATCHFORD, D. 1979. Exploitation of the Green Iguana. The Herptile 4(4):37-39.

112. BOLTON, M. 1978. Crocodule farming in Papua New Cumea Oryx 14(4):365-369 (see also Editors note, p 36y)

113 BURY, R. B. 1979. Review of the ecology and conservation of the Bog Turtle, Clemmys muhlenbergii. US. Fish \& Wildl. Special Scientific Report-Wildlife No. 219.

114. CIFUENTES, M. \& MACFARLAND, C. 1978. Galapagos Islands (Ecuador). WWF Yearbook 1977-1978:139-146. (Project Reports involving Tortoises, Land Iguanas and Sea Turtles)

115. COGGER, H.G. 1974. Voyage of the Banded Iguana. Austral.Nat.Hist. 18(4):144-149.

116. ERNST, C.H., \& GILROY, M.J. 1979. Are LeatherbackTurtles, Dermochelys coriacea, common along the Middle Ätlantic Coast? Bull.Md.Herp.Soc. 15(1):16-19.

117. FELGER, R.S. 1979. Project 1471. Gulf of California, Mexico-Marine Turtles. WWF Yearbook, 1978-1979:168-169.

118. FITCH, H.S. \& HENDERSON, R.W. 1977. Age and Sex Differences, Reproduction and Conservation of Iguana iguana. Contribs. in Biol. \& Geol. Milwaukee Public Museum, No. 13:I-21. 
119. FOOTE, R.W. 1978. Nesting of Podocnemis unifilis (Testudines: Pelomedusidae) in the Colombian Amazon Herpetologica 34(4):333-339.

120. FRAZIER, J-G. 1979. Marine Turtle Management in Seychelles: A Case-study. Environmental Conserv. 6(3):225-230.

121. FRETEY, J. 1976. Les tortues mannes de Guyane française. Courrier de la Nature. No. 41:10-21.

122. FRETEY, J. 1977. Causes de mortalıté des Tortues luths adultes (Dermochelys coriacea) sur le littoral guyanais. Courrier de la Nature. No. 52:257-266.

123. GELDIAY, R. 1979. Project 1419. Marine Turtles-Conservation along coast of Turkey. WWF Yearbook 1978-1979: 139-141.

124. GIBSON, T.S.H. 1979. Green Turtle (Chelonia mydas (L.)) nesting activity at Aldabra Atoll. Phil.Trans.R.Soc.Lond.B. 286:255-263.

125 HARDY, G.S., \& WHITAKER, A.H. 1979. The status of New Zealand's endemic reptles and their conservation. Forest and Bird. No. 214. 13(4):34-39.

126. HATHAWAY, R.R. 1972. Sea Turtles and unanswered questions about Sea Turtles in Turkey. (Turkish \& English texts). Balik ve Balıkillk ("Fish \& Fisheries") 20(1):1-8. EBK Balıkfilk Müessesesi, Müdürlüğü

127. HIGAREDA, G.P. 1979. Morelet's Crocodile (Crocodylus moreleti Dumeril \& Dumeril), in the region of Los Tuxtlas Veracruz, Mexico, Bull.Md.Herp.Soc. 15(1):20-21.

128. HIRTH, H.F., \& ABDEL LATIF, E.M. 1980. A nesting colony of the Hawksbill Turtle Eretmochelys imbricata on Seil Ada Kebir Island, Suakin Archipelago, Sudan. Biol.Conserv. 17(2): 125-130.

129. IVERSON, J.B. 1978. The impact of feral cats and dogs on populations of the West Indian Rock Iguana, Cyclura carinata. Biol.Conserv. 14(1):63-73.

130. JENKINS, R.W.G. 1978. The Status of Endangered Australian Reptiles, Chapter 16, pp.169-176, in, TYLER, M.J. (Ed.) The Status of Endangered Australasian Wildlife. Adelaide: Royal Zool. Soc. South Australia

131. LAMBERT, M.R.K. 1979. Trade and the Mediterranean Tortoises, Oryx 15(1):81-82.

131. LIMPUS, C. J. \& McLACHLAN, N.C. 1979. Observations on the Leatherback Turtle, Dermochelys coriacea (L.), in Austraila. Austral. Wildl.Res. 6(1):105-116.

133. MCDIARMID, R.W. (Ed.) 1978. Amphibians and Reptiles, Vol. 3, in PRITCHARD, P.C.H. (Ed.) Rare and Endangered Biota of Florida. Gainesville: University Presses of Florida.

134. MEDEM, F. 1976. Project 748. Orinoco Crocodile-Status Survey. WWF Yearbook 1975-1976.

135. MITTERMEIER, R.A. 1978. South America's River Turtles: saving them by use. Oryx 14(3):222-230.

136. MOLL, E. O. 1977. Project 1313. River Terrapin. WWF Yearbook 1976-1977:136-138.

137. OGDEN, J.C. 1978. Status and nesting biology of the American Crocodile, Crocodylus acutus, (Reptilia, Crocodilidae) in Florida. J.Herpetol. 12(2): 183-196.

138. REIDY, P. 1980. Swan song for Westem Australian tortoise? Herpetofauna 11(2):2-4

139. ROSS, J.P. 1979. Project 1320. Marine Turtle Survey (Oman), WWF Yearbook 1978-1979:109-111.

140. SCHLEICH, H-H. 1979. Der Kapverdische Riesenskink, Macroscincus coctei, eine ausgestorbene Echse? Natur und Museum 109(5):133-138.

141. SHAFFER, J.C., \& ERNST, C.H. 1979. The Giant Land Tortoises of Aldabra, Geochelone gigantea. Bull.Md.Herp.Soc. 15(2): 46-55.

142. SINGH, V.B. 1978 (1979). The status of Gharial (Gavialis gangeticus) in U.P. and its rehabilitation J.Bombay Nat.Hist.Soc. 75(3):668-683.

143. SMITH, N.J.H. 1979. Aquatic Turtles of Amazonia: an endangered resource. Biol.Conserv. 16(3):165-176.

144. TROTTER, M. (Ed.). 1978. Proceedings of 1978 Symposium, Desert Tortoise Council. (Previous Symposia 1976 \& 1977) D.T.C. 1835 Klauber Ave., San Diego, Calif.92114.

145. VARONA, L.S. 1980. Protection in Cuba. Oryx 15(3):282-284.

146. WALCZAK, P.S. 1979. The status of marine turtles in the waters of the Yemen Arab Republic. Brit.J.Herpetol. 5:851-853.

147 WELLS, G. 1979. Crocodiles in Australia. Tigerpaper (FAO).6(1):19-22.

148. WHITAKER, R. 1978. Common Indian Snakes. Macmillan (India) Ltd

149. WHITĀKER, R. \& DANIEL, R.C. 1978. The status of Asian crocodilians. Tigerpaper (FAO). 5(4):12-17.

150. WHITAKER, Z \& WHITAKER, R. 1979. Never a smile for a crocodile. Anim.Kingd. Oct.-Nov::31-34.

151. ZWINENBERG, A.J. 1979. Biologie en status van de Levantijnse Adder van de Cycladen, Vipera lebetina schweizeri. Lacerta 37(9):137-152.

152. GROOMBRIDGE, B, 1982. The IUCN Amphibia-Reptilia Red Data Book. Part 1. Testudines, Crocodylia, Rhynchocephalia. IUCN, Gland, Switzerland.

153. GIBBONS, J.R.H. 1981. The biography of Brachylophus (Iguanidae) including the description of a new species B.vitiensis from Fiji. J. Herpetol. 15(3): 255-273. 


\section{(B) Major taxonomic sources utilised}

(see notes $\mathrm{p} 8$ ).

ARNOLD, E.N. 1973. Relationships of the Palaearctic Lizards assigned to the genera Lacerta, Algyroides and Psammodromus (Reprilia: Lacertidae). Bull. Br. Mus. nat. Hist. (Zool.)25(8):291-366.

BOHME, W. and KLAVER, C.J.J. 1980. The systematic status of Chamaeleo kinetensis Schmidt, 1943, (Sauria Chamaeleonidae) from the Imantong Mountains, Sudan, with comments on lung and hemipenial morphology within the C. bitaeniatus group. Amphibia-Reptilia 1:3-17

BOUR, R. 1978. Les tortues actuelles de Madagascar (République malgache): liste systématique et description de deux sousespèces nouvelles (Reptilia-Testudines). Bull.Soc.Et.sci.Anjou N.S. 10:141-154

BRAME, $\bar{A} . \mathrm{H} .1970$. A new species of Batrachoseps (Slender salamander) from the desert of souther California Contr.Sci.No. 200:1-11.

BRYGOO, E.R. 1969. Chamaeleo guentheri Boulenger, 1888, synonyme de C.pardalis Cuvier, 1829 Bull.Mus.natn.Hist.nat.Paris. Ser. 2, 41(1):119-121

BRYGOO, E.R., BLANC, C.P. \& DOMERGUE, C.A. 1972. Notes sur les Chamaeleo de Madagascar. 10. Deux nouveaux caméléons des hauts sommets de Madagascar: C. capuroni n.sp. et C. gastrotaenia andringitraensis n subsp. Bull.Mus natn.Hist.nat.Paris. Ser. 2, 42:601-613.

BRYGOO, E.R., BOURGAT, R., \& DOMERGUE, C.A. 1972. Notes sur les Chamaeleo de Madagascar, C. tuzetae n.sp., nouvelle espèce du sud-ouest (Reptilia, Squamata, Chamaeleonidae). Bull.Mus.natn.Hist.nat.Paris. Ser. 3. $21: 133-140$.

BRYGOO, E.R., BLANC, C.P., \& DOMERGUE, C.A. 1974. Notes sur les Chamaeleo de Madagascar XII Caméléons due Marojezy. C. peyrieresi n.sp. et C. gastrotaenia guillaumetin. subsp. (Reptilia, Squamata, Chamaeleonidae). Bull.Acad.malgache. 51(1):151-166.

BRYGOO, E.R, \& DOMERGUE, C.A. 1967(1968), Description d'un Caméléon nouveau de Madagascar, Chamaeleo tsaratananensis n.sp. Bull.Mus.natn.Hist.nat.Panis. Ser. 2, 39(5):829-832.

BRYGOO, E.R., \& DOMERGUE, C.A. 1968. Les Caméléons a rostre impair et rigide de l'ouest de Madagascar. Mém.Mus.natn.Hist.nat.Paris. N.S. Ser. A, 52(2):1-110.

BRYGOO, E.R., \& DOMERGUE, C. A. 1969. Chamaeleo balteatus Dum et Bib. (dans C. et A. Duméril, 1851) n'est pas synonyme de C. bifidus Brongniart, 1800. Bull.Mus.natn.Hist.nat.Paris. Ser. 2, 41(1):104-116.

BRYGOO, E.R., \& DOMERGUE, C.A. 1970. Notes sur les Chamaeleo de Madagascar. C. belalandaensis n.sp., Caméléon du Sud-Ouest. Bull.Mus.natn.Hist.nat.Paris. Ser. 2, 42(2):305-310.

CAREY, M.W. 1975. The Rock Iguana, Cyclura pinguis, on Anegada, British Virgm Islands, with notes on Cyclura ricordi and Cyclura comuta on Hispaniola. Bull.Fla.State Mus.Biol.Sci. 19(4):189-234.

CHEKE, A.S. 1975. An undescribed Gecko from Agalega: Phelsuma agalegae sp. nov. Bull. Mauritius Inst. 8(1):33-48.

COGGER, H. 1979. Reptiles and Amphibians of Australia. Sydney: A.H. \& A.W. Reed PTY. Ltd. 2nd Edn. COLLINS, J.T., HUHEEY, J.E., KNIGHT, J.L., \& SMITH, H.M. 1978. Standard Common and Current Scientific Names for North American Amphibians and Reptiles. Society for the study of Amphibians and Reptiles, Herpetological Circular No. 7:1-36.

DOWLING, H.G. \& GIBSON, F.W. 1970. Relationship of the Neotropical Snakes Hydrodynastes bicinctus and Cyclagras gigas. Herpetol. Rev.2(2):37-38.

DUELLMAN, W.E. 1975. On the classification of Frogs. Occ.Pap.Mus.nat.Hist.Univ.Kansas. No. 42:1-14. FLEMING, R.L. Jr. and FLEMING, R.L. Sr. 1973. Some Snakes from Nepal. J. Bombay Nat.Hist.Soc. 70(3):426-437. FUNK, R.S. 1979. Python reticulatus in East Pakistan (= Bangladesh); correction of an erroneous record. Bull.Chicago Herp.Soc. 14(3):92.

GANS, C. \& WILLIAMS, E.E. 1954. Present knowledge of the snake Elachistodon westemanni Reinhardt. Breviora No. 36 .

GIBBONS, J.R.H. 1981. The biogeography of Brachylophus (Iguanidae) including the description of a new species, B.vitiensis from Fiji. J. Herpetol. 15(3):255-273.

GORHAM, S.W. 1974. Checklist of World Amphibians up to January 1, 1970. New Brunswick: The New Brunswick Museum

GOW, G.F. 1971. A new species of Python from Åmhem Land. The Australian Zoologist. 19:133-139.

GOW, G.F. 1981. A new species of Python from Central Australia. Aust.J.Herp. 1(1):29-34.

GRANDISON, A.G.C. 1978. The occurrence of Nectophrynoides (Ānura, Bufonidae), in Ethiopia. A new concept of the genus with a description of a new species. Monitore zool. Ital. (N.S.) Suppl. XI, 119-172.

HEYER, W.R., \& LIEM, O.S. 1976. Analysis of the Intergeneric Relationships of the Australian Frog Family Myobatrachidae. Smithsonian Contrib Zool. No. 233.

KLEMMER, K. 1963. Liste der rezenten Giftschlangen. Elapidae, Hydrophiidae, Viperidae und Crotalidae, pp. 255-464 in Die giftschlangen der Erde, Marburg: Behringwerk-Mitt. Spec. Suppl.

LAMOTTE, M., \& PERRET, J.L. 1968. Revision du genre Conraua Nieden. Bull. de l'I.F.A.N. Ser. A., 30:1603-1644.

LIEM, S.S. 1970. The morphology, systematics, and evolution of the Old World Treefrogs (Rhacophoridae and Hyperoliidae). Fieldiana Zool. 57.1-145.

LOVERIDGE, A. \& WILLIAMS, E.E. 1957. Revision of the African Tortoises and Turtles of the Suborder Cryptodira. Bull.Mus.comp. Zool.Harvard, 115(6):163-557.

LYNCH, J.D. 1973. The transition from Ârchaic to Advanced Frogs. Chap. 3 in, VIAL, J.L. (Ed.) Evolutionary

Biology of the Anurans, Columbia: Univ. of Missouri Press. 
MCDIARMID, R.W. 1971. Comparative Morphology and Evolution of Frogs of the Neotropical Genera Ätelopus, Dendrophryniscus, Melanophryniscus and Oreophrynells. Bull. Los Angeles Co. Mus.nat.Hist.Sci. 12:1-66. McDOWELL, S.B. 1964, Partition of the genus Clemmys and related problems in the taxonomy of the aquatic Testudinidae. Proc.zool.Soc.Lond. 143(2):239-279.

McDOWELL, S.B. 1975. A Catalogue of the snakes of New Guinea and the Solomons, with special reference to those in the Bernice P. Bishop Museum. Part 2. Anilioidea and Pythoninae. H.Herpetol. 9:1-79.

McDOWELL, S.B. 1979. A Catalogue of the snakes of New Guinea and the Solomons, with special reference to those in the Bernice P. Bishop Museum. Part 3. Boinae and Acrochordoidea (Reptlia, Serpentes) J.Herpetol. 13:1-91.

MERTENS, R. 1963. Helodermatidae, Varanidae, Lanthanotıdae. Das Tierreich 79:1-26, Berlin: Walter de Gruyter. MERTENS, R. 1966a. Die nichtmadagassischen Arten und Unterarten der Gekkonengattung Phelsuma.

Senck.biol.47(2):85-110.

MERTENS, R. 1966b. Chamaeleonidae. Das Tierreich 83:1-37. Berlin. Walter de Gruyter.

MERTENS, R. 1966c. Ein neuer Zwergwaran aus Australien. Senck.biol.47:437-441.

MERTENS, R. 1968. Zur Kenntnis der Herpetofauna von Kamerun und Femadno Poo. Bonn.zool.Beitr.19:69-84.

MERTENS, R. 1970. Neues über einige Taxa der Geckonengattung Phelsuma. Senck.biol. 51(1/2):1-13.

MILLER, T. J. \& SMITH, H. M. 1980. The Lesser African Rock Python. Bull.Md Herp.Soc. 15(3):70-84.

MONTANUCCI, R.R. 1970. Analysis of Hybridisation between Crotaphytus wislizenii and Crotaphytus silus

(Sauria, Iguanidae) in California. Copeia 1970(1):104-123.

OBST, F.J. 1980. Ergänzende Bemerkungen zu den Testudiniden Madagaskars (Reptlia، Chelonia, Testudinidae) Zool.Abh.Mus.Tierk. Dresden 36(12):229-232.

PACE, A.E. 1974. Systematic and biological studies of the Leopard Frogs (Rana pipiens complex) of the United States. Misc.Publ.Mus.Zool.Univ.Mich. 148:1-140.

PERRET, J.L. 1971. Les espèces du genre Nectophrynoides d'Afrique (Batraciens, Bufonidés). Annls.Fac.Sci.

Cameroun No. 6:99-109.

PERRET, J.L. 1972. Les espèces des genres Wolterstorfina et Nectophrynoides d'Årique. Annls.Fac.Sci.

Cameroun No. 11:93-119.

PETERS, J.A. \& DONOSO-BARROS, R. 1970. Catalogue of the Neotropical Squamata; Part 2, Lizards and

Ämphisbaenians. Bull. U.S. natn.Mus. 297(2):1-293.

PETERS, J.A. \& OREJAS-MIRANDA, B. 1970. Catalogue of the Neotropical Squamata; Part 1, Snakes. Bull. U.S. natr.Mus. 297(1):1-347.

PRESCH, W. 1973. A review of the Tegus, Lizard genus Tupinambis (Sauria, Teiidae) from South America.

Copeia 1973(4):740-746.

PRITCHARD, P.C.H. 1979. Encyclopedia of Turtles. Hong Kong: T.F. H. Publications Inc., Ltd.

RHODIN, A.G.J., MITTERMEIER, R.A., GARDNER, A.L., \& MEDEM, F. 1978. Karyotypic analysis of the

Podocnemis turtles, Copeia 1978(4):723-728.

ROSSMAN, D.A. \& EBERLE, W.G. 1977. Parttion of the genus Natrix, with Preliminary Observations on

Evolutionary trends in Natricine Snakes, Herpetologica 33(1):34-43.

RUSSELL, A.P. 1977. The genera Rhoptropus and Phelsuma (Reptlia: Gekkonidae) in southem Africa: a case of convergence and a reconsideration of the biogeography of Phelsuma.Zool.Africana 12(2):393-408.

SCHWARZ, A., \& CAREY, M. 1977. Systematics and evolution in the West Indian iguanid genus Cyclura.

Studies on the Fauna of Curacao and other Caribbean Islands. No. 73, Utrecht.

SCHWARZ, A., \& THOMAS, R. 1975. A check-list of West Indian Amphibians and Reptiles. Camegie

Mus.nat.Hist. Spec. Publ. No. 1:1-216.

SHEPLAN, B.R. \& SCHWARZ, A. 1974. Hispaniolan Boas of the genus Epicrates (Serpentes, Boidae) and their Antillean relationships. Annls. Cargegie Mus. 45(5):57-143.

SMITH, H.M., \& JAMES, L.F. 1958. The Taxonomic Significance of Cloacal Bursae in Turtles. Trans. Kansas

Acad.Sci. 61(1):86-96.

SMITH, L.A. 1981 . A revision of the genera Aspidites and Python (Serpentes: Boidae) in Western Australia.

Rec.West Aust.Mus, 9(2):211-226.

STIMSON, A.F. 1969. Boidae. Das Tierreich 89:1-49. Berlin: Walter de Gruyter.

STULL, O.G. 1935. A check-list of the family Boidae. Proc.Boston.Soc.nat.Hist. 40:387-408.

UNDERWOOD, G. 1954. On the classification and evolution of Geckos. Proc.zool.Soc.Lond. 124(3):469-492.

UNDERWOOD, G. 1976. A systematic analysis of boid snakes. In, Bellairs, A. d'A., \& Cox, C.B. (Eds.)

Morphology and Biology of Reptiles: 151-175. Linnean Society Symposium Series No. 3. London: Academic Press.

VANZOLINI, P.E., \& VALENCIA, J. 1965. The genus Dracaena, with a brief consideration of macroteild relationships (Sauria, Teiidae). Arquivos de Zoologia 13:7-35.

VINSON, J., \& VINSON, J.M. 1969. The saurian fauna of the Mascarene Islands. Bull.Mauritius Inst. 6(4):203-320. WERMUTH, H. 1965. Gekkonidae, Pygopodidae, Zantusiidae. Das Tierreich 80:1-246. Berlin: Walter de Gruyter. WERMUTH, H. 1967. Agamidae. Das Tierreich 86:1-127. Berlin: Walter de Gruyter

WERMUTH, H. 1968. Cordylidae (Cordylnae + Gerrhosaurinae). Das Tierreich 87:1-30. Berlin: Walter de Gruyter. WERMUTH, H. \& FUCHS, K. 1978. Bestimmen von Krokodilen und ihrer Häute. Stuttgart: Gustav Fischer. 1-100. WERMUTH, H., \& MERTENS, R. 1977. Testudines, Crocodylia, Rhynchocephalia. Das Tierreich 100:1-174.

Berlin: Walter de Gruyter.

WILLIAMS, E.E. 1954. New or redescribed pelomedusid skulls from the Tertiary of Africa and Asia

(Testudines, pelomedusidae). Breviora (Harvard) No. 39:1-8. 
XAVIER, F. 1978. Une espèce nouvelle de Nectophrynoides (Annoure, Bufonidae) des Monts Nimba. N. liberiensis n.sp. Bull.Soc.zool.Fr. 103(4):431-441.

(C) Miscellaneous, including general crocodile and marine turtle references.

ANON (PARKER, H.W.). 1933. The collection of Reptile skins for commercial purposes. (Report by the Advisory Committee on Hides and Skins). South Kensington, London: Imperial Institute BRAZAITIS, P. 1973. The identufication of living crocodilians. Zoologica (NY). 58(4):59-101.

DODD, C.K. 1979. A bibliography of endangered and threatened amphibians and reptiles in the U.S. and its terntones (conservation, distribution, natural history, status). Smithsonian Herpetological Information Service, No. 46:1-35. (Washington, D.C.)

FUCHS, K. 1974. Die Krokodilhaut. Darmstadt: Eduard Roether Verlag

HOOGMOED, M.S. 1977. De handel in reptielehuiden in Singapore. Lacerta 35(5):66-74. (inc. English summary). INSKIPP, T., \& WELLS, S. 1979. Intemational trade in Wildlife. Earthscan (10 Percy St., London W IP 0DR). KING, F.W., \& BRAZAITIS, P. 1971. Species identification of commercial crocodile skins. Zoologica (NY) $56(2): 15-70$

MACK, D., DUPLAIX, N., \& WELLS, S. 1979. Intemational Trade in Sea Turtle Products (The Sea Turtle: an animal of divisible parts). Traffic (International) \& Traffic (USA). Revised edition, 1980

MROSOVSKY, N. (Ed.) Marine Turtle Newsletter. (Depts, of Zoology \& Psychology, Univ, of Toronto, Ontario, Canada M5S 1 Al).

WERMUTH, H. \& FUCHS, K. 1978. Bestimmen von Krokodilen und ihrer Häute. Stuttgart: Gustav Fischer. WOOD, D. A. 1977. A bibliography of the World's rare, endangered, and recently extinct wildlife and plants Environmental Ser. No.3. Environmental Inst., Oklahoma State Univ

WORLD CONFERENCE ON SEA TURTLE CONSERVATION. Nov, 1979, Washington D.C --a symposium volume on the Conference is anticipated in 1981. Among reports on the Conference is: CHERFAS, J, 1979. The song of the turtle. New Scientist 84 (1185) 880-882: 
A baco Island Boa 46

Abbott's Day Gecko 28

Achoque I1

(Acinixys) 20

Acrantophis 44

Adanson's Turtle 23

Afghan Tortoise 20

African Chameleon 32

African Large-grain Lizard 42

African Python 50

African Rock Python 50

African Savanna Monitor 42

African Sharp-nosed Crocodile 26

African Slender-snouted Crocodile26

African Small-grain Lizard 43

Agra Lizard 40,41

Agra Monitor 42

(Agrionemys) 19

Alabama Red-bellied Turtle 17

Aldabra Giant Tortoise 18

\section{Alligator 25}

Almeda Striped Racer 52

Armargosa Toad 14

Amblyrhynchus 37

Ambon Lizard 42

Ambystoma 11

Ameiva 40

American Alligator 25

American Crocodile 26

Amethystine (Rock) Python 49

Ampalague 43

Anaconda 48

Andaman Day Gecko 29

\section{Andrias l 1}

Anegada Ground Iguana 38

Angolan Python 49

Angonoka 18

Angulated Tortoise 17

Aniella 41

Annulated Tree Boa 45

Anolis 37

Aquatic Box Turtle 17

Araramboia 45

Archey's Frog 13

Areolated Tortoise 19

Argentine Teju 40 (two entries)

Argentine Tortoise 17

Armenian Viper 53

Arrau 24

Aruba Island Rattlesnake 53

Asiatic Cobra 51

Aspidites 44

(Asterochelys) 18

Atelopus 14

Atlantic Ridley 21

Atlantic Saltmarsh Snake 52

Australian Fresh-water Crocodile 26

Axolotl 11

\section{Baby Python 47}

Baghdad Small-grain Lizard 41

Bahama Islands Boa 46

Ball Python 49

Banded Tegu 40
Barred Python 48

Barrington Land Iguana 37

Batagur 16

Batrachophrynus 13

Batrachoseps 11, 12

Baw-baw Frog 13

Beach Cobra 52

Beaded Lizard 41

Bell's Hinged Tortoise 19

Bengal Lizard 40,41

Bengal Python 49

Berlandier's Tortoise 18

Black Caiman 25

Black-headed Python 44

Black Legless Lizard 41

Black Pond Turtle 16

Black Side-necked Turtle 23

Black Soft-shell Turtle 22

Black Spiny-tailed Lizard 30

Black Toad 14

Blood Python 49

Blunt-nosed Leopard Lizard 38

Blunt-nosed Viper 53

Blunt-tailed Sand Boa 47

Boa 44

(Boa) 45,46

Boa Constrictor 44

Boelen's Python 49

Bog Turtle 16

Bolson Tortoise 19

Bolyeria 45

Borneo Lizard 42

(Bothrochilus) 48

Bothrops 53

Boulenger's Tortoise 19

Bowsprit Tortoise 17

Brazil Teju 39

Broad-nosed Caiman 25

Broad-snouted Crocodile 26

Brazllian Giant Tortoise 17

Brachylophus 37

Bufo 14

Burmese Brown Tortoise 18

Burmese Python 49

Burmese Starred Tortoise 18

Burmese Swamp Turtle 17

\section{Cabragoya 42}

Caiman 25

Caiman Lizard 40

Calabaria 45

Calabar Ground Python 45

Calcutta Lizard 41

California Tiger Salamander 11

Callagur 16

Cameroon Toad 14

Candoia 45

Cape Clawed Toad 13

Cape Platanna 13

Cape Verde Giant Skink 41

Caretta 21

Carettochelys 22

Carpet Python 50

Casarea 45
Cat Island Turtle 17

Caymans Ground Boa 50

Central American River Turtle 16

Central Asian Cobra 53

Central Assian Monitor 41

Chaco Sideneck Turtle 24

Chaco Tortoise 17

Chamaeleo 31-37

Charina 45

Cheat Mountain Salamandar 12

Chelonia 21

Chersina 17

Child rens (Rock) Python 48

Chinese Alligator 25

Chinese Giant Salamander 11

Chondropython 45

Chioglossa 11

(Chrysemys) 17

Clelia 51

Clemmys 16

Cnemidophorus 40

Common Batagur 16

Common Chameleon 33

Common Hinged Tortoise 19

Common Iguana 38

Common Monitor 41

Common Tegu 40

Common Tortoise 19

Conolophus 37,38

Conraua 15

(Constrictor) 43

Corallus 45,46

Cordylus 39,40

Coromandel Leiopelma 13

Creaser's Mud Turtle 16

Crested Chameleon 33

Crested Lizard 30

Crocodilurus 40

Crocodylus 26,27

Croco-Teju 40

Crotalus 53

(Crotaphytus) 38

Cuatro Cienegas Soft-shell Turtle 22

Cuban Tree Boa 46

Cuban Crocodile 27

Culebra Giant Anole 37

Cuvier's Smooth-fronted Caiman 25

(Cyclagras) 50

Cyclura 38

Cyrtodactylus 28

D'Alberti's Python 48

Dahl's Toad-headed Turtle 24

(Damonia) 16

Dark Soft-shell Turtle 22

Day Geckos 28-30

Deep Springs Toad 14

Dermatemys 16

Dermochelys 22

Desert Chameleon 35

Desert Monitor 42

Desert Pygmy Monitor 42

Desert Slender Salamander 11

Desert Tortoise 19 
Diamond Python 49, 50

Discoglossus 13

Dracaena 40

Drymarchon 52

Dumerl's Boa 44

Dumeril's Monitor 42

Dunn's Mud Turtle 16

Dura Turtle 16

Dwarf Caiman 25

Dwarf Monitor 43

Eastern Indigo Snake 52

Egyptian Spiny-tailed Lizard 31

Egyptan Tortoise 20

Elachistodon 50

Eleutherodactylus 13

Emerald Monitor 43

Emerald Tree Boa 45

(Enygrus) 45

Epicrates 46,47

Eretmochelys 21

Erymnochelys 23

Eryx 47

Estuarine Crocodile 26

Eunectes 47,48

Eurycea 12

(Eurycea) 12

Exiliboa 48

False Gavial 27

False Gharial 27

Fiji Banded Iguana 37

Fiji Crested Iguana 37

Fiji Snake 53

Filfola Lizard 39

Fischer's Chameleon 33

Fischer's Tree Boa 47

Fish Lizard 42

Flap-necked Chameleon 33

Flatback Turtle 2]

Flattened Musk Turtle 16

Florida Gopher Tortoise 19

Flower Lizard 42

Ford's Boa 46

Forest Hinged Tortoise 19

Forest Tortoise 17

Forked Chameleon 34

Four-horned Chameleon 36

Freckled Monitor 44

Furrowed Wood Turtle 17

Gaboon Turtle 23

Galapagos Giant Tortoise 18

Galapagos Land Iguana 38

Galapagos Marine Iguana 37

\section{Gallotia 39}

Gambelia 38

Ganges Soft-shell Turtle 22

Garden Tree Boa 46

Gardiner's Seychelles Frog 15

Gavialis 27

Gavial 27

Geoclemys 16

Geochelone 17, 18
(Geoemyda) 16

Geometric Tortoise 20

Gerrhonotus 41

Gharial 27

Giant Ânole 37

Giant Garter Snake 52

Giboya 43

(Gigantorana) 15

Gila Monster 41

Golden Coqui Frog 13

Golden Frog 14

Golden Toad 14

Gold-dust Day Gecko 29

Gold-striped Salamander 11

Goliath Frog 15

Gongylomorphus 41

(Gongylophis) 47

Gopherus 19

Gould's Monitor 42

Graceful Chameleon 34

Gray's Monitor 42

Greek Tortoise 20 (two entries)

Green Iguana 38

Green Tree Python 45

Green Turtle 21

Grey Monitor 4]

Grooved Tortoise 18

Grotto Salamander 12

(Gymnodactylus) 28

Haitian Ground Boa 51

Haitian Vine Boa 46

Hamilton's Frog 13

Hardwick's Spiny-tailed Lizard 31

Hawksbill Turtle 21

Helmeted Turtle 23

Heloderma 4l

Heosemys 16

Hercules Tortoise 17

Hermann's Tortoise 20

Hierro Giant Lizard 39

Hispaniola Ground Iguana 38

Hochstetter's Frog 13

Hoge's Sideneck Turtle 24

Home's Hinged Tortoise 19

Homopus 19

Horn Lizard 3 ]

Horn Lizard Small-grain 31

Horsfield's Tortoise 20

Houston Toad 14

Hydrodynastes 52

Hydromantes 12

Hydrosaurus 30

\section{Hyla 15}

\section{Igruana 38}

Illnois Chorus Frog 15

Illinois Mud Turtle 54

Impressed Tortoise 18

Inagua Island Turtle 17

Indian Egg-eating Snake 52

Indian Flap-shell Turtle 22

Indian Monitor 41

Indian Oval-grain Lizard 42
Indian Python 49 (two entries)

Indian Sand Boa 46

Indian Sawback Turtle 16

Indian Small-grain Lizard 40

Indian Soft-shell Turtle 22

Indian Star or Starred Tortoise 18

Indian Tent Turtle 16

Irapuca 23

Island Night Lizard 30

Israel Painted Frog 13

Italıan Spadefoot Toad 13

Jaboti 17

Jacaré 24

Jacarura 39

Jackson's Three-homed Chameleon 34

Jamaica Boa 47

Jamaica's Ground Iguana 38

Japanese Giant Salamander [1

Java Lizard 42

Java Rock Python 50

Java Tree Python 49

Javelin 47

Jayakar's Sand Boa 47

Jemez Mountains Salamandar 12

Johnson's Crocodile 26

Kachuga 16

Karoo Tortoise 19

Kavalai Forest Turtle 16

Kemp's Ridley 21

Kenya Sand Boa 46

Kem Canyon Slender Salamander 11

Kinixys 18,19

Kinosternon 16,54

(Klauberina) 31

Komodo Dragon 43

Kuhl's Tortoise 19

Lace Monitor 44

(Lacerta) 39

Lake Erie Water Snake 52

Lake Junin (Giant) Frog 13

Lake Lerma Salamander 11

Lake Patzcuaro Salamander 11

Larch Mountain Salamander 12

Latufi's Viper 53

Leatherback 22

Leathery Turtle 22

Lebetune Viper 53

Leiopeima 13

Leiolopisma 41

Leopard Tortolse 18

Lepidochelys 21

Lesser African Rock Python 50

Levantune Vıper 53

Liasis 48

(Liasis) 48,49

Lichanura 48

Limestone Salamander 12

Lissemys 22

Loggerhead Turtle 2]

Long-tailed Rock Monitor 42

Loxocemus 49 
Luth 22

Macabe Forest Skink 41

Macroscincus 41

Madagascar Boa 44

Madagascar Day Gecko 29

Madagascar Flat-shelled Tortoise 20

Madagascar Tortoise 18

Madagascar Tree Boa 50

Magdalena River Turtle 24

Maja 45

Malachochersus 19

Malayan Monitor 43

Mangrove Monitor 42,43

Margined Tortoise 20

Marsh Crocodile 26

Masacuate 43

Masticophis 52

Mastigures 30

Maurtius Greater Day Gecko 28

Mediterranean Chameleon 33

Megalixalus 15

(Megalsbatrachus) 11

Melanochelys 16

Melanosuchus 25

Meller's Chameleon 35

Merten's Water Monitor 43

Mexican Giant Gopher Tortoise 19

Mexican Spotted Wood Turtle 17

Mindoro Crocodile 26

Mitchell's Water Monitor 43

Molurus Python 49

Mona Blind Snake 44

(Morelia) 49

Morelet's Crocodile 26

Morenia 17

Mountain Chameleon 35

Mountain Tortoise 18

Mount Nimba Viviparous Toad 14

Moumful Tree Monitor 44

Mugger 26

Muhlenberg's Turtle 16

Mulga Monitor 16

Müller's Sand Boa 47

Musurana 52

\section{Naja 53}

(Nardoana) 48

Narrow-bridged Mud Turtle 16

(Natrix) 52

Nectophrynoides 14,15

Nerodia 52

Nesomantis 15

New Guinea Crocodile 26

New Guinea Plateless Turtle 22

New Mexico Ridge-nosed

Rattlesnake 51

(Nicoria) 16

Nile Crocodile 26

Nile Monitor 43

Nile Soft-shell Turtle 22

No-mark Lizard 42

North Island Leiopelma 13
Oedura 28

Ogmodon 53

Olive Ridley 21

Olive (Rock) Python 48

Olm 12

Orange-throated Whiptail 40

Orange Toad 14

Oriental Rock Python 49

Orınoco Crocodile 26

\section{Osteolaemus 27}

Oustalet's Giant Chameleon 35

Owen's Three-horned Chameleon 35 Red-headed Sideneck Turtle 24

Oxus Cobra 53

Pacific Boa 45

Pacufic Monitor 42

Pacific Ridley 21

Painted Terrapin 16

\section{Paleosuchus 25}

Palm Lizard 30

Panamint Alligator Lizard 41

Pancake Tortoise 19

Papuan Ground Boa 45

Panther Chameleon 36

Papuan Monitor 43

Papuan Python 48

\section{Paradelma 30}

Parrot-beaked Tortoise 19

Parson's Grant Chameleon 36

Peacock-marked Softshell Turtle 22

Pelobates 13

Pelomedusa 23

Peltocephalus 23

Pelusios 23

Penang Lizard 40

Perentie 42

\section{Phaeognathus 12}

Phelsuma 28-30

Philippines Crocodule 26

Philippine Lizard 42

Philoria 13

Phrynops 24

Phrynosoma 39

Pigmy Mulga Monitor 31

Pine Barrens Tree Frog 15

Platypus Frog 14

Plain Lizard 42

Platemys 24

Plethodon 12

Plymouth Red-bellied Turtle 17

Podarcis 39

Podocnemis 23, 24

(Podocnemis) 23

Proteus 12

Psammobates 20

Pseudacris 15

Pseudernydura 24

Pseudemys 16, 17

(Pseudoboa) 51

Pseudocordylus 40

Puerto Rican Boa 46

Pygmy Mulga Monitor 42

Python 49-50

Pyxis 20
Queensland Snake-Lizard 30

Queimada Island Bothrops 53

Radıated Tortoise 18

Rainbow Boa 46

Ramgodie 42

Rana 15

(Rana) 15

Red-foot Tortolse 17

Red-headed Amazon Turtle 23

Red Hills Salamander 12

Red Teju 40

Relict Leopard Frog 15

Reticulated Python 50

Reticulated Velvet Gecko 28

(Rhampholeon) 35

Rheobatrachus 14

Rhinoclemmys 17

(Rhoptropella) 29

Rice Lizard 40, 42

Ricord's Ground Iguana 38

Ridge-nosed Rattlesnake 53

Ridge-tailed Monttor 43

Ring Lizard 42

Rio Apaporis Caiman 25

River Terrapin 16

Rodriguez Day Gecko 30

Roofed Turtle 16

Rosy Boa 48

Rough-necked Monitor 43

Rough-scaled Carpet Snake 49

Round Island Boa 45

Round Island Day Gecko 29

Round Island Keel-scaled Boa 45

Round Island Skink 41

Royal Python 49

Rubber Boa 45

Rusty Monitor 43

Saigon Python 49

Sail-fin Lizard 30

Salt-water Crocodile 26

Sand Boa 47

Sand Monitor 42

San Diego Horned Lizard 39

San Esteban Island Chuckwalla 39

San Francisco Garter Snake 52

San Joaquin Leopard Lizard 38

San Joaquin Coachwhip 52

San Marcos Salamander 12

Santa Cruz Long-toed Salamander 11

Sanzinia 49

Sauromalus 39

Schneider's Smooth-fronted Caiman25

Schweigger's Hinged Tortoise 19

Semi-Java Lizard 42

Senegal Chameleon 36

Sepik Monitor 43

Serpent Island Gecko 28

Serrated Tortoise 20
Rhinoceros Iguana 38 
Seychelle Islands Tree Frog 15

Seychelles Frog 15

Shasta Salamander 12

Short-homed Chameleon 32

Short-necked Swamp Turtle 24

Short-tailed Dwarf Chameleon 35

Short-tailed Pygmy Monitor 41

Short-tailed Python 49

Siam Lizard 42

Siamese Crocodile 27

Side-striped Day Gecko 29

Singapore Lizard 42

Slender Chameleon 37

Slender-Snouted Crocodile 26

Soa-Soa Water Lizard 30

Socotra Chameleon 35

Solomons Ground Boa 45

Sonoran Green Toad 14

\section{Sooglossus 15}

South American 'Chameleon' 38

South American Red-lined Turtle 17

South American River Turtle 24

South American Water 'Cobra' 52

Speckled Tortoise 19

Spectacled Caiman 25 (two entries)

Spencer's Monitor 43

\section{Sphenodon 27}

Spider Tortoise 20

Spiny Chameleon 36

Spiny-tailed Lizard 30

Spiny-tailed Pygmy Monitor 41

Spix's Sideneck Turtle 24

Spotted Pond Turtle 16

Spotted Sand Boa 47

Spotted Tree Monitor 44

Spurred Tortoise 18

Spur-thighed Tortoise 20

St. Croix Ground Lizard 40

Stephen Island Leiopelma 13

\section{Sternotherus 16}

Steak-tailed Monitor 41

Stripe-backed Side-necked Turtle 23

Sulawesi (Celebes) Tortoise 18

Sumatra Lizard 42

Sururucu 52

Talagoya 40

Tartary Sand Boa 47

Tegu 39

Tehachapi Slender Salamander 12

Tent Tortoise 20

Teracay 24

Terrapene 17

Testudo 20

(Testudo) 17, 18, 20

Texas Blind Salamander 12

Texas Gopher Tortoise 18

\section{Thamnophis 52}

Thomasset's Seychelles Frog 15

Three-keeled Land Tortoise 16

Timor Python 50

Timor Tree Monitor 43

Tomistoma 27

Toothed Cape Tortoise 20

\section{Tracajá 24}

Trachyboa 50

Transcapian Desert Monitor 4

Transcaucasian Long-nosed Viper 53

Travancore Tortoise 18

Tree 'Crocodile' 43

Tree Lizard 42

Trionyx 22

Tropidophis 50, 51

Tuatara 27

Tuntong 16

Turks \& Caicos Ground Iguana 38

Tupinambis 40

Two-banded Chameleon 32

Two-striped Garter Snake 52

Typhlomolge 12

Typhlops 44

Typhlotriton

Ungaliophis 51

Uromastyx 30, 31

Varanus 41-44

Variegated Dwarf Chameleon 36

Vegas Valley Leopard Frog 15

Vipera 51,53

Viviparous African Toad 14

Water Box Turtle 17

Water Monitor 43

Water Python 48

West African Dwarf Crocodile 27

West African Python 50

West Australian Rock Python 48

Western Gopher Tortoise 19

Western Swamp Turtle 24

White-breasted Side-necked Turtle 23

Woma 44

Xantusia 30

Xenoboa 51

Xenopus 13

Yellow Anaconda 48

Yellow-foot Tortoise 17

Yellow Monitor 42

Yellow-spotted Amazon Turtle 24

Yellow Tortoise 18

Zetek's Golden Frog 14 
Lithographed by The Nuffield Press Limited, Cowley, Oxford, England. 

The Nature Conservancy Council is the government body which promotes nature conservation in Great Britain. It gives advice on nature conservation to governmentandall whose activities affect our wildlife and wild places. It also selects, establishes and manages a series of National Nature Reserves. This work is based on detailed ecological research and survey. Its Great Britain headquarters are at: Nature Conservancy Council 19/20 Belgrave Square London SWIX 8PY United Kingdom.

This is one of a range of publications produced by the NCC. A catalogue listing current titles is available from Interpretative Branch Nature Conservancy Council Attingham Park Shrewsbury Shropshire SY4 4TW United Kingdom.

\section{Ag NATURE CONSERVANCY COUNCIL}

ISBN 0861392248 @ NCC 1983 Produced by Interpretative Branch 